\title{
ELEMENTARY REACTIONS AND THE INTERPRETATION OF MEASUREMENTS OF CHEMICALLY REACTING NON L.T.E. PLASMAS
}

\author{
H. W. DRAWIN \\ Association EURATOM-CEA sur la Fusion, Département de Physique du Plasma et de la Fusion Contrôlée, Centre \\ d'Etudes Nucléaires, Boîte Postale No. 6, 92260 Fontenay-aux-Roses, France
}

\begin{abstract}
The "thermodynamic state" and the spatial-temporal evolution of the composition of chemically reacting plasmas are generally described by the Boltzmann or Pauli kinetic equations (balance equations for the transfer of particle densities, momentum, and energy for the different chemical species). Due to the numerous difficulties encountered when solving these coupled differential equations, simplifying assumptions are often introduced. The results therefore depend, on the one hand, on the elementary reactions chosen for the model calculations and, on the other hand, on the cross-sections. Vice versa, the interpretation of both the thermodynamic state and the diagnostic measurements is dependent on the kind of elementary reactions introduced in the model.

The dependence on the different atomic and molecular elementary reactions of the atomic (molecular) models adopted is demonstrated for some cases such as hydrogen, helium, highly ionized atoms, nitrogen, on the basis of a Maxwellian velocity distribution. As a refined step, deviations from a Maxwellian distribution should be taken into account. Especially treated are: (a) Excited state populations; (b) Calculation of recombination and ionization coefficients; (c) Influence of deviations from a Maxwell distribution of the electrons; (d) Influence of collisions between chemically excited particles.

The laser is often used for plasma diagnostic purposes. The influence of the plasma state by a superimposed laser radiation field is briefly treated including photo-ionization and recombination stimulated by one and two laser photons. Omission of these reactions can lead to erroneous interpretation of measurements. Some of the model calculations are compared with experimental results.
\end{abstract}

\section{INTRODUCTION}

Chemically reacting plasmas represent special types of ionized gases in which several technical and scientific disciplines are interested. Plasma chemical processes are widely applied in industry for producing thin inorganic (such as $\mathrm{TiO}, \mathrm{TiO}_{2}, \mathrm{SiO}_{2}, \ldots$ ) or organic films on special substrates. Plasma chemical reactions can be applied to produce pure carbides such as $\mathrm{TiC}, \mathrm{TaC}, \mathrm{Ta}_{2} \mathrm{C}, \mathrm{WC}$, $\mathrm{W}_{2} \mathrm{C}, \ldots$ It is possible to extract from plasma chemical reactors very pure metals such as $\mathrm{Ti}, \mathrm{Si}, \mathrm{Ta}, \mathrm{Mo}, \mathrm{W}, \ldots$ or even pigments such as $\mathrm{TiO}_{2}$ (the latter being of importance in the colour industry). It is also possible to produce in a plasma, acetylene $\mathrm{C}_{2} \mathrm{H}_{2}$, ozone $\mathrm{O}_{3}$, hydrogenperoxide $\mathrm{H}_{2} \mathrm{O}_{2}$, hydrazine $\mathrm{N}_{2} \mathrm{H}_{4}$, hydrogen cyanide $\mathrm{HCN}$, and many other chemical substances. Much effort is presently expended in order to find conditions under which production rates are optimized. Many research laboratories and industrial firms are working on chemical media which, under plasma conditions, yield population inversion and permit lasing of those media. Examples are the development of the $\mathrm{HCN}, \mathrm{HF}, \mathrm{CO}_{2}, \mathrm{CO}, \mathrm{N}_{2}$, and the rare gas laser systems. The light industry undertakes research to find new plasma chemical systems with special emission properties. A large research activity is being developed to understand the different types of plasma chemical reactions which have led to the formation of the earth's ionosphere. Physicists working on thermonuclear fusion are also interested in a special type of "chemical plasma", since plasma chemical reactions in the plasmawall region of the fusion reactor proper, and in the divertor $\dagger$ seem to have an influence on the properties of the hot plasma core.

†Special device for extracting impurity ions from a fusion plasma. The problem is to neutralize the ions and to fix the neutrals on the wall.
Although the temperature and densities of all these plasmas are extremely different they have one common property, namely that they shown significant deviations from thermodynamic equilibrium. A quantitative understanding of these chemically reacting plasmas can only be obtained by studying their non-equilibrium properties. This is of special importance in connection with the interpretation of diagnostic measurements and the optimization of reaction rates.

At present we are still far away from a detailed understanding even of the simplest non-equilibrium systems such as a recombining hydrogen or helium plasma. In this short review I will show how physicists have dealt with non L.T.E. plasma properties in order to interprete measurements made in such plasmas. For reasons of completeness it seems useful to begin with a brief description of the state of thermodynamic equilibrium followed by a summary of the kinetic equations describing the non equilibrium state. The latter shall then be applied to selected plasma-chemical situations.

\section{EQUILIBRIUM AND NON-EQUILIBRIUM}

From a thermodynamic point of view plasmas can be divided into three different classes, namely those which are:

1-in complete thermodynamic equilibrium (C.T.E.),

2-in local thermodynamic equilibrium (L.T.E.),

3-neither in complete nor in local thermodynamic equilibrium. We call them briefly "non L.T.E. plasmas".

It is this third class of plasmas which we will deal with. But before going into detail I would like to make some general comments about the three classes of plasmas in order to show the difficulties we will meet when treating non-equilibrium properties of plasmas. 
In complete thermodynamic equilibrium, the plasma state is entirely determined by pressure and temperature: the temperature $T$ suffices to calculate the relative chemical composition of the gas via the mass action laws. These are the Guldberg-Waage equation for dissociation-recombination equilibrium of type

$$
(\mathrm{AB}) \rightleftarrows(\mathrm{A})+(\mathrm{B})
$$

for the chemical constituents (AB), (A) and (B). The equation has the form

$$
\frac{n_{\mathrm{A}} n_{\mathrm{B}}}{n_{\mathrm{AB}}}=\frac{P_{\mathrm{A}}(T) P_{\mathrm{B}}(t)}{P_{\mathrm{AB}}(T)}\left(\frac{m_{\mathrm{A}} m_{\mathrm{B}}}{m_{\mathrm{AB}}}\right)^{3 / 2} \frac{(2 \pi k T)^{3 / 2}}{h^{3}} \exp \left(-\frac{\mu_{\mathrm{AB}}}{k T}\right)
$$

where $n$ denotes the number density, $P(T)$ the temperature-dependent partition function, $m$ the mass, $T$ the temperature, $\mu_{\mathrm{AB}}$ the reaction energy, and $k$ and $h$ are Boltzmann's and Planck's constants respectively. Equation (1) also holds for dissociation ionizationrecombination of type

$$
(\mathrm{AB}) \rightleftarrows\left(\mathrm{A}^{+}\right)++\left(\mathrm{B}^{-}\right)
$$

For ionization-recombination equilibrium of type

$$
\left(\mathrm{A}^{r+}\right) \rightleftarrows\left(\mathrm{A}^{(r+1)+}\right)+(e)
$$

(with $r=$ stage of ionization) the mass action law is known as Saha's equation and takes the form

$$
\begin{aligned}
\frac{n_{r+1} n_{e}}{n_{r}}= & \frac{P_{r+1}(T) P_{e}(T)}{P_{r}(T)}\left(\frac{2 \pi m_{e} k T}{h^{2}}\right)^{3 / 2} \\
& \times \exp \left(-\frac{E_{r, r+1}-\Delta E_{r, r+1}}{k T}\right)
\end{aligned}
$$

where $E_{r, r+1}$ is the ionization energy for the transfer of ionization stages $r \rightarrow r+1$, and $\Delta E_{r, r+1}$ is the lowering of the ionization energy due to Coulomb interaction of charged particles. The partition function $P_{e}(T)$ of the electrons is a constant: $P_{e}(T)=2$. Saha's equation can also be applied to reactions in which a negative ion is formed as a result of electron attachment as for instance in the following reaction:

$$
\left(\mathrm{A}^{0}\right)+e \rightleftarrows\left(\mathrm{A}^{-}\right)
$$

This reaction is of greatest importance in electric circuit breakers in which highly electro-negative gases are employed. In this case, the Saha equation is

$$
\frac{n_{0} n_{e}}{n_{-}}=\frac{P_{0}(T) 2}{P_{-}(t)}\left(\frac{2 \pi m_{e} k T}{h^{2}}\right)^{3 / 2} \exp \left(\frac{E_{0,-}}{k T}\right)
$$

where $E_{0,-}$ is the "electron affinity" (attachment energy).

The number of "mass action equations" is generally equal to the number of chemical constituents present minus two; thus, the number of equations is indeed sufficient for calculating the relative constants or number densities which one adds a conservative equation to the relative number densities. The absolute number densities can be determined by the equation of state which relates total pressure, total absolute particle densities and absolute temperature. I would like to stress that the computation of chemical equilibrium compositions is mathematically based on the solution of a system of algebraic equations, in contrast to the calculation of non-equilibrium compositions.

In practice, computation of chemical compositions is often not as easy as it seems at a first glance. Apart from computational difficulties there is still a significant lack of many atomic and molecular data essential for calculating the partition functions entering into the mass action laws. Besides this, principal problems such as the determination of the lowering of ionization energy and of the cut-off level of bound electronic states are not yet definitely solved. At present, only little is known about the existence of negative ions, their possible bound states and the electron affinities.

Conditions for establishing complete thermodynamic equilibrium-which require constant temperature and pressure across the whole system, with no temporal variations-are seldomly fulfilled. In almost all cases experimental constraints cause more or less strong departures from homogeneity or from the stationary state and, thus, from complete thermodynamic equilibrium distributions; in other words: the thermodynamic state is influenced by the external constraints. If however, the gradients and temporal variations are not too large and if the densities are sufficiently high, the equilibrium relations describing the distribution over the various chemical constituents may still be verified locally. This is the state of local thermodynamic equilibrium which refers to the local kinetic temperature of the interacting particles. This implicitly means that all species have a Maxwellian velocity distribution for a common local temperature $T$, and that the distribution over the bound energy states is Boltzmannian relative to the local temperature.

If measurements of inhomogeneous or transient plasmas are interpreted on the basis of equilibrium relations one must realize that their validity is restricted. Moreover, the precision of such an interpretation highly depends on:

- the atomic and molecular data which serve for evaluating the measurements,

- the atomic and molecular data necessary for calculating the partition functions and, thus, the equilibrium concentrations,

- the way in which the experimental constraints have been taken into account.

I would like to emphasize that even in this very classical field of equilibrium thermodynamics of plasmas, important work has still to be done in order to refine our knowledge about chemical plasma compositions. Equilibrium considerations remain in any case useful also when nonequilibrium problems are treated, since one gets an idea about the chemical composition towards which the non-equilibrium plasma evolves.

Non L.T.E. plasmas generally can not be described by the above mentioned equilibrium relations; the interpretation of measurements of such plasmas and the calculation of the thermodynamic non-equilibrium state has to be based on elementary reactions considerations. In addition to the already mentioned difficulties in the case of L.T.E. plasmas, we are now faced with new problems which demand detailed knowledge about

- the nature and type of elementary reactions which are important under the special non L.T.E. conditions considered,

-the cross-sections of these reactions as a function of collision energy,

-the distribution function of particle velocities and the photon field. 
Indeed, the physical properties and chemical compositions of plasmas depend in any case on the individual reactions. In equilibrium they just yield the well-known equilibrium relations described by the mass action law. In thermodynamic non-equilibrium the macroscopic properties are described by the Boltzmann or Pauli kinetic equations. They replace the equilibrium relations when the non L.T.E. state of plasmas is treated. The Pauli equations can be considered as velocity space-integrated Boltzmann equations. They yield statistical averages of the plasma parameters like the equilibrium relations but admit deviations from the equilibrium distributions due to the individual collision and radiation processes.

\section{FUNDAMENTAL KINETIC EQUATIONS IN PLASMA SYSTEMS}

To begin with, let us briefly recall the basic statistical equations by which non-equilibrium (or non L.T.E.) plasma can be described. More detailed information may be found in Refs. 1-9. For pure Coulomb systems, see Ref. 10.

In the following we distinguish different chemical species by subscripts $k, l \ldots$, and excited bound states by a second subscript $h, i, j, \ldots$ Electrons are characterized by subscript $e$. Different ionization stages of the same chemical element are considered as "different chemical species" in the present context. Further, $w$ is the velocity vector, $\langle w\rangle=(1 / n) \int_{w} n(w) d w$ is the mean velocity vector with respect to the laboratory system, $n$ the number density.

We have the following transfer equations for chemical species $k$ in bound state $i$ (see Ref. 8):

For the rate of change of particle densities $n_{k, i}$

$$
\frac{\partial n_{k, i}}{\partial t}+\nabla_{r} \cdot\left(n_{k, i}\left\langle\mathbf{w}_{k, i}\right\rangle\right)=\left(\frac{\partial n_{k, i}}{\partial t}\right)_{\substack{\text { collision } \\ \text { radiation }}} .
$$

For the rate of change of momentum $n_{k, i} m_{k}\left\langle\mathbf{w}_{k, i}\right\rangle$

$$
\begin{aligned}
\frac{\partial\left(n_{k, i} m_{k, i}\left\langle\mathbf{w}_{k, i}\right\rangle\right)}{\partial t}+ & \nabla_{r} \cdot\left(n_{k, i} m_{k}\left\langle\mathbf{w}_{k, i}\right\rangle\left\langle\mathbf{w}_{k, i}\right\rangle\right)-n_{k, i} \mathbf{F}_{k, i} \\
& =\left(\frac{\partial\left(n_{k, i} m_{k}\left\langle\mathbf{w}_{k, i}\right\rangle\right)}{\partial t}\right)_{\substack{\text { collision } \\
\text { radiation }}}
\end{aligned}
$$

where

$$
n_{k, i} \mathbf{F}_{k i}=n_{k, i} q_{k, i}\left(\mathbf{E}+\frac{1}{c}(\mathbf{j} \times \mathbf{B})\right)
$$

is the generalized Lorentz force $(\mathbf{E}=$ electric field strength, $\mathbf{B}=$ magnetic induction, $\mathbf{j}=$ current density, $q_{k, i}=$ electric charge of particle considered).

For the rate of change of kinetic energy $n_{k, i}(1 / 2) m_{k}\left(w_{k, i}\right)^{2}$ :

$$
\begin{aligned}
& \frac{\partial}{\partial t}\left(n_{k, i}\right.\left.\frac{1}{2} m_{k}\left\langle w_{k, i}\right\rangle^{2}\right)+\nabla_{r} \cdot\left(n_{k} \frac{1}{2} m_{k}\left\langle\mathbf{w}_{k, i}\right\rangle^{2}\left\langle\mathbf{w}_{k, i}\right\rangle\right) \\
&-n_{k, i} \mathbf{F}_{k, i} \cdot\left\langle\mathbf{w}_{k, i}\right\rangle=\left(\frac{\partial n_{k}(1 / 2) m_{k}\left\langle w_{k, i}\right\rangle^{2}}{\partial t}\right)_{\substack{\text { collision } \\
\text { radiation }}} .
\end{aligned}
$$

For the rate of change of internal energy $E_{k, 1 i}$ :

$$
\frac{\partial}{\partial t}\left(n_{k, i} E_{k, 1 i}\right)+\nabla_{r} \cdot\left(n_{k, i} E_{k, 1 i}\left\langle\mathbf{w}_{k, i}\right\rangle\right)=\left(\frac{\partial n_{k, i} E_{k, 1 i}}{\partial t}\right)_{\substack{\text { collision } \\ \text { radiation }}}
$$

$E_{k, 1 i}$ is the internal energy (excitation energy) of species $k$ in excited states $i$, relative to the ground state 1 . When the particles are in higher ionization stages, one has to add the ionization energies to the excitation energies.

The first term on each of the left-hand sides describes temporal changes, the second term spatial changes due to diffusion in coordinated space, the right-hand sides are generalized expressions for the rate of change due to collisional and radiative effects. They contain sources and sinks. It seems worth mentioning that eqn (8) for the rate of change of internal energy is generally not explicitly written. One only considers eqn (7) for the particle kinetic energies, and at the end one adds a heat flux vectorwhich accounts for diffusion of internal energy-and a radiation term which accounts for loss or gain of internal energy due to the escape or capture of photon energy. This method has for instance been adopted in Ref. 8 .

In plasmas the radiation field can considerably contribute to the change of particle densities and of internal energy, whereas momentum transfer due to photonparticle interaction can in most cases be neglected. Thus, we have to add corresponding transfer rate equations. Consider photons of frequency $\nu$ traveling with velocityc in the direction of unit vector s. The photon velocity vector is then given by $\mathbf{w}=c \mathbf{s}$. Denoting the specific radiation intensity in the frequency interval $\nu \ldots \nu+\mathrm{d} \nu$ by $I_{\nu}(\mathbf{s})$ the photon number distribution (i.e. the number of photons crossing unit cross-section in unit time per unit solid angle) is given by $f_{\nu}(\mathbf{s})=I_{\nu}(\mathbf{s}) / h \nu$. The Boltzmann equation for $f_{\nu}(\mathbf{s})$ becomes

$$
\frac{\partial}{\partial t} f_{\nu}(\mathbf{s})+\nabla_{r} \cdot\left(f_{\nu}(\mathbf{s}) c \mathbf{s}\right)=\left(\frac{\partial f_{\nu}(\mathbf{s})}{\partial t}\right)_{\substack{\text { collision } \\ \text { radiation }}} .
$$

When the dependency of light velocity upon particle density is omitted $c$ is a constant. Thus, eqn (9a) takes the simplified form: ${ }^{11}$

$$
\frac{\partial}{\partial t} f_{\nu}(\mathbf{s})+c \mathbf{s} \cdot \nabla_{r} f_{\nu}(\mathbf{s})=\left(\frac{\partial f_{\nu}(\mathbf{s})}{\partial t}\right)_{\substack{\text { collision } \\ \text { radiation }}} .
$$

For instance, for a plasma containing the chemical species $k$ with only two possible bound states $i$ and $j$ (and which lead to radiative emission and absorption $i \rightleftarrows j$ ) the right-hand side of eqns $(9 a, b)$ has the form $(j$ is the higher, $i$ the lower of the two states):

$$
\begin{aligned}
\left(\frac{\partial f_{\nu}(\mathrm{s})}{\partial t}\right)_{\substack{\text { collision } \\
\text { radiation }}} & =c\left(-n_{k, i} \mathrm{~B}_{k, i j} \phi_{k, i j}(\nu) f_{\nu}(\mathrm{s})\right. \\
& \left.+n_{k, j}\left(\frac{1}{4 \pi} \dot{\mathrm{A}}_{k, j i} \eta_{k, j i}(\nu)+\mathrm{B}_{k, j i} \psi_{k, j i}(\nu) f_{\nu}(\mathbf{s})\right)\right)
\end{aligned}
$$

$A_{k, j i}, B_{k, j i}$ and $B_{k, i j}$ are the Einstein coefficients for spontaneous emission, induced emission and absorption respectively within transition $i \rightleftarrows j$ of chemical species $k . \phi(\nu), \eta(\nu)$ and $\psi(\nu)$ are the normalized line profiles for absorption, spontaneous emission and induced emission respectively:

$$
\int_{\text {line }} \phi(\nu) \mathrm{d} \nu=1 ; \quad \int_{\text {line }} \eta(\nu) \mathrm{d} \nu=1 ; \quad \int_{\text {line }} \psi(\nu) \mathrm{d} \nu=1 .
$$

Equation (10) does not contain contributions from radiative diffusion which should additionally be taken into account if necessary. 
Equations (9b) and (10) can be combined to give the radiative transport equation for specific intensity $I_{\nu}(\mathrm{s})=$ $f_{\nu}(\mathbf{s}) h \nu$ in direction s:

$$
\begin{aligned}
\frac{\partial I_{\nu}(\mathbf{s})}{c \partial t}+\mathbf{s} \cdot \nabla_{r} I_{\nu}(\mathbf{s}) & =\epsilon_{\nu}-\kappa_{\nu}^{\prime} I_{\nu}(\mathbf{s}) \\
& =\kappa_{\nu}^{\prime}\left(S_{\nu}-I_{\nu}(\mathbf{s})\right)
\end{aligned}
$$

where $\epsilon_{\nu}$ and $\kappa_{\nu}^{\prime}$ are the frequency-dependent local emission and effective absorption coefficients respectively, and $S_{\nu}=\epsilon_{\nu} / \kappa_{\nu}^{\prime}$ is the radiative source function.

The equation for the transfer rate of photon energy, i.e. the change of radiation energy per unit volume and unit time, is obtained by multiplying eqn (9b) by $h \nu$ and then integrating over frequency $\nu$ and solid angle $\Omega$. One obtains $^{11}$

$$
\frac{\partial u_{\mathrm{rad}}}{\partial t}+\nabla_{r} \cdot \mathbf{J}=\left(\frac{\partial}{\partial t} \int_{\nu=0} \int_{\Omega} I_{\nu}(\mathrm{s}) \mathrm{d} \nu \mathrm{d} \Omega\right)_{\substack{\text { collision } \\ \text { radiation }}}
$$

where the local radiation density $u_{\mathrm{rad}}$ is given by

$$
u_{\mathrm{rad}}=\frac{1}{c} \int_{\nu=0} \int_{\Omega} h \nu f_{\nu}(\mathrm{s}) \mathrm{d} \nu \mathrm{d} \Omega
$$

and the local radiative vector flux by

$$
\mathbf{J}=\int_{\nu=0}^{\infty} \int_{\Omega} s I_{\nu}(\mathbf{s}) \mathrm{d} \nu \mathrm{d} \Omega
$$

The right-hand side of eqn (12) is a generalized radiative source term which accounts for creation and destruction of photon energy flux due to collisional-radiative processes. As can be seen from eqn (10), this term depends on the particle densities.

The transfer rate eqns $(4,5,7)$ and $(8)$ represent velocity-space integrated equations. In order to calculate the terms appearing on the right-hand sides one has to know the velocity distributions of the interacting particles. Since it is a difficult task to determine them one often assumes that they are Maxwellian, and since the electrons are of ten much more energetic than the heavy particles one makes the simplifying assumption that the electrons obey a Maxwellian distribution according to an electron temperature $T_{e}$, whereas the heavy particles obey a Maxwell distribution belonging to a heavy particle temperature $T_{h}$. These assumptions are not always justified. For instance, the electron velocity distribution function can considerably deviate from a Maxwellian one when the electrons are submitted to strong externally applied electric or magnetic fields. Deviations from a Maxwell distribution can also occur as a result of inelastic and superelastic electron-atom, electron-molecule and electron-ion collisions. Radiative processes can have a considerable influence on the distribution function. The latter can also be changed by strong spatial density and temperature gradients.

The electron velocity distribution function $f_{e}(\mathbf{w})$ is governed by the Boltzmann equation

$$
\frac{\partial f_{e}}{\partial t}+\mathbf{w}_{e} \cdot \nabla_{r} f_{e}+\frac{1}{m_{e}} \mathbf{F}_{e} \cdot \nabla_{w} f_{e}=\left(\frac{\partial f_{e}}{\partial t}\right)_{\substack{\text { collision } \\ \text { radiation }}}
$$

where $f_{e} \mathrm{dw} \equiv f_{e}(\mathbf{r}, t, \mathbf{w}) \mathrm{dw}$ is the number density of electrons (at spatial position $r$ at time $t$ ) which have velocity vectors lying between $w$ and $w+d w$. The electron density $n_{e}(\mathbf{r}, t)$ is obtained by integrating over all velocities:

$$
n_{e}(\mathbf{r}, t)=\int_{0}^{\infty} f_{e}(\mathbf{r}, t, \mathbf{w}) \mathrm{dw} .
$$

Equation (15) has been treated by many authors for different experimental situations, see e.g. Refs. 12-46. The large number of publications during recent years shows that there is a high research activity in this field. In fact, the knowledge of the electron distribution function is of vital importance in the interpretation of many measurements especially in the case of plasma of low degree of ionization.

It has been shown experimentally ${ }^{48-51}$ and theoretically ${ }^{52-57}$ that the heavy particle distributions show deviations from a Maxwellian one especially after the gas or plasma has expanded through an orifice into a vacuum, or into a region of low pressure. In this case a special flow field is established which is called a free jet expansion. Far enough ahead from the orifice the flow becomes supersonic within a few orifice diameters. The forces exerted on the molecules and atoms parallel and perpendicular to the flow direction are so different that the distribution function becomes non isotropic. According to Hamel and Willis ${ }^{53}$ the velocity distribution of a monatomic chemically non-reacting gas can be approximated by the so-called ellipsoidal distribution function. The ellipsoidal model allows the local distribution function $f$ to be made separable in terms of Gaussian distribution functions with distinct parallel and perpendicular local temperatures $T_{\|}$and $T_{\perp}$ respectively. $f$ has the following analytical form:

$$
\begin{aligned}
f=n\left\{\left(\frac{m}{2 \pi k T_{\|}}\right)^{1 / 2} \exp (\right. & \left.\left.-\frac{m\left(w_{\|}-\left\langle w_{\|}\right\rangle\right)^{2}}{2 k T_{\|}}\right)\right\} \\
\times & \left\{\left(\frac{m}{2 \pi k T_{\perp}}\right) \exp \left(-\frac{m w_{\perp}^{2}}{2 k T_{\perp}}\right)\right\}
\end{aligned}
$$

where

$$
\begin{aligned}
\left\langle w_{\|}\right\rangle & =\frac{1}{n} \iint_{-\infty}^{+\infty} \int w_{\|} f \mathrm{~d}^{3} w \text { (mean flow velocity) } \\
T_{\|} & =\frac{1}{n k} \int_{-\infty}^{+\infty} \iint\left(w_{\|}-\left\langle w_{\|}\right\rangle\right)^{2} f \mathrm{~d}^{3} w \text { (parallel temperature) } \\
T_{\perp} & =\frac{1}{n k} \int_{-\infty}^{+\infty} \iint w_{\perp}^{2} f \mathrm{~d}^{3} w \text { (perpendicular temperature). }
\end{aligned}
$$

Not much is known about the distribution functions of molecular gases. Since the populations of rotational and vibrational states of molecules depend primarily on the collision frequency between heavy particles, eventual deviations from Maxwellian distributions are of greatest importance for understanding plasma-chemical reactions in expanding gases.

The following conclusions may be drawn from this section: The non-equilibrium state of chemically reacting plasmas is governed by a system of differential and integro-differential equations which have to be solved in a self-consistent manner. Due to the large number of individual collisions and radiation processes it appears to be a hopeless task to solve all equations simultaneously and to find self-consistent solutions. Many simplifying assumptions are generally introduced in order to explain as well as possible the essential features of non- 
equilibrium states under special experimental situations. To find the appropriate assumptions is of greatest importance in connection with the interpretation of diagnostic measurements of non-equilibrium plasmas. This is sometimes a difficult task, since one has to know the most important reactions. Our knowledge in the field is still rather scanty for many chemical systems.

In order to make the problems mathematically tractable one applies simplified reaction models. They are based on idealized reaction schemes for the elementary reactions. I will now show how the latter intervene in the interpretation of measurements and in the calculation of the composition of chemically reacting non L.T.E. plasmas. Whereas the plasma chemist is mainly interested in macroscopic results and is happy when he succeeds in finding new ways for producing new substances, the physicist wants to know why the reactions occur just in this manner and not in another way. Non-equilibrium systems treated by the chemist are still too difficult for the physicist who prefers systems as simple as possible. It will turn out that even in these cases big mistakes can be made in the establishment of reaction schemes.

\section{POPULATIONS OF EXCITED STATES IN QUASI-HOMOGENEOUS, QUASI-STATIONARY PLASMAS WITH MAXWELLIAN ELECTRON} VELOCITY DISTRIBUTION

\section{Helium}

Consider a quasi-homogeneous, quasi-stationary non L.T.E. state. For reasons of simplicity, let us consider a helium plasma at low densities and sufficiently high temperature. Thus, helium molecules and molecular ions can be neglected. The electrons are assumed to have a Maxwellian distributions. In thermal equilibrium the excited levels have a Boltzmann population relative to each other. The electron temperature $T_{e}$ can then be determined from the intensity ratio of two spectral lines originating from levels with different excitation energies. This is a classical diagnostic method. (For spectroscopic diagnostic methods, see e.g. Ref. 58.)

Under non-equilibrium situations the upper state populations may strongly deviate from a Boltzmann population, the actual population densities depend on the excitation and de-excitation mechanisms. They change with plasma composition. Due to the very different energy dependence of the excitation cross-section $\sigma$ for singlet and triplet states (see Fig. 1) the ratio of triplet to singlet states populations might be expected to depend strongly on electron temperature. The same temperature dependence is then obtained for the ratio of two spectral lines belonging to the triplet and singlet system respectively. In 1955 , Cunningham ${ }^{59}$ proposed to determine electron temperatures $T_{e}$ from the intensity ratio of such two spectral lines. Since then, the method has often been applied. I will show now that the application of this method can lead to an incorrect interpretation of measured spectral line intensities.

Consider the two lines $\lambda=4713 \AA\left(4^{3} \mathrm{~S}-2^{3} \mathrm{D}\right)$ and $\lambda=4921 \AA\left(4^{1} \mathrm{D}-2^{1} \mathrm{P}\right)$. For an equilibrium plasma the line intensity ratio is practically temperature independent, since the energy difference of the upper state populations is nearly zero, see curve L.T.E. in Fig. 2.

We assume now that the two upper levels are directly populated by electronic collisions from the ground level ${ }^{1} \mathrm{~S}_{0}$, whereas depopulation occurs through spontaneous de-excitation only. All other processes are neglected. The rate eqns (4) for the number densities $n_{4 \mathrm{~S}}, n_{4 \mathrm{D}}$ take the special

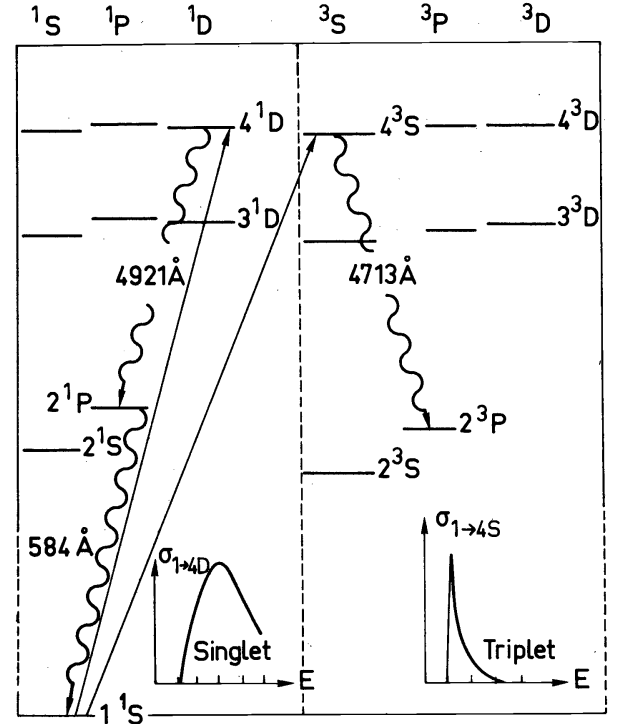

Fig. 1. Level system of atomic helium and energy dependence of electron excitation cross-sections for singlet and triplet states.

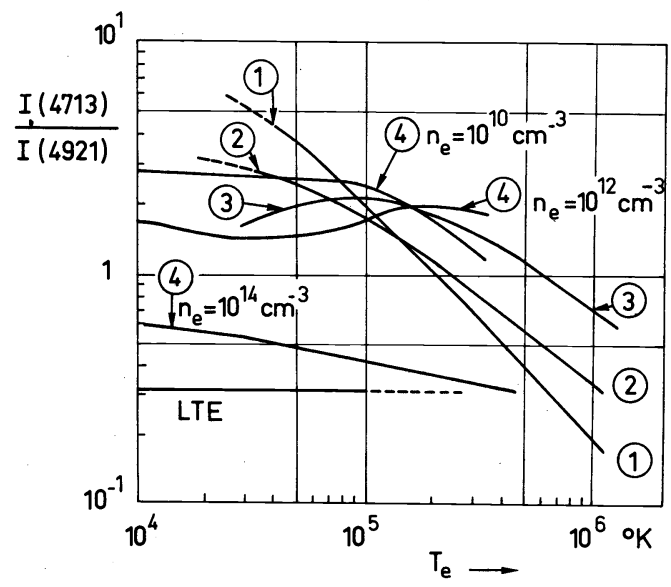

(1) Direct excitation without coupling (Lees)

(2) Direct " " " (St. John)

(3) Coupling, heavy particle collisions within multiplicities $\left(n_{1 S}=10^{12} \ldots .10^{14} \mathrm{~cm}^{-3}\right)$.

(4) Coupling, electron collisions within and between multiplicities; optically thin

Fig. 2. Calculated intensity ratio $I(4713) / I(4921)$ after Drawin and Henning. ${ }^{61}$ Meaning of curves: L.T.E. = Local thermodynamic equilibrium; (1)=Coronal model with excitation cross sections after Lees (1932); (2) = Coronal model with excitation cross sections after St. John et al. ${ }^{60}(1964)$; (3) = Coupling between excited levels within each multiplicity due to heavy particle collisions included; (4)= Complete collisional radiative model. All curves refer to optically thin He plasma.

form

$$
\begin{gathered}
\frac{\partial n_{4 \mathrm{~S}}}{\partial t}+\nabla_{r} \cdot\left(n_{4 \mathrm{~S}}\left\langle\mathbf{w}_{4 \mathrm{~S}}\right\rangle\right)=\mathrm{C}_{1 \mathrm{~S} \rightarrow 4 \mathrm{~S}} n_{e} n_{1 \mathrm{~S}}-n_{4 \mathrm{~S}} \sum_{h<4} \mathrm{~A}_{4 \mathrm{~S} \rightarrow h} \\
\frac{\partial n_{4 \mathrm{D}}}{\partial t}+\nabla_{r} \cdot\left(n_{4 \mathrm{D}}\left\langle\mathbf{w}_{4 \mathrm{D}}\right\rangle\right)=\mathrm{C}_{1 \mathrm{~S} \rightarrow 4 \mathrm{D}} n_{e} n_{1 \mathrm{~S}}-n_{4 \mathrm{D}} \sum_{h<4} \mathrm{~A}_{4 \mathrm{D} \rightarrow h}
\end{gathered}
$$

where $\mathrm{C}_{1 \mathrm{~S} \rightarrow 4 \mathrm{~S}, 4 \mathrm{D}}$ are the temperature-dependent excitation coefficients (calculated with a Maxwellian velocity dis- 
tribution)and the A terms are the Einstein coefficients. For a quasi-homogeneous, quasi-stationary state the terms on the left-hand sides are equal to zero. Thus, one obtains two algebraic equations for $n_{4 \mathrm{~S}}$ and $n_{4 \mathrm{D}}$. For the ratio of the line intensities the expression is then

$$
\frac{\mathrm{I}(4713)}{\mathrm{I}(4921)}=\frac{\mathrm{A}_{4713} h \nu_{4713}}{\mathrm{~A}_{492 !} h \nu_{4921}} \cdot \frac{\sum \mathrm{A}_{4 \mathrm{D} \rightarrow h}}{\sum \mathrm{A}_{4 \mathrm{~S} \rightarrow h}} \cdot \frac{\mathrm{C}_{1 \rightarrow 4 \mathrm{~S}}}{\mathrm{C}_{1 \rightarrow 4 \mathrm{D}}} .
$$

With the excitation cross-sections of Lees measured in 1932 one obtains curve 1 of Fig. 2 . With the more recent cross-sections of St. John et al. ${ }^{60}$ curve 2 is obtained. The intensity ratio is indeed temperature-dependent as expected, the precision of this ratio depends on the cross-sections for the elementary processes considered.

The $4^{3} \mathrm{~S}$ and $4^{1} \mathrm{D}$ levels are not isolated from the other levels. Collisions take place among all excited levels and also between the excited levels and the continuum of free electrons. Also photo-excitation and ionization processes can occur. One should therefore expect a mixing of the excited state populations and, thus, an influence on the populations of the $4^{1} \mathrm{D}$ and $4^{3} \mathrm{~S}$ levels. In order to demonstrate this effect quantitatively we will now add to the direct electronic excitation processes from the ground state $1^{1} \mathrm{~S}$ heavy particle collisions between helium ground state atoms and excited atomic species. We take only into account transfer reactions within each multiplicity. As may be seen from curve 3 of Fig. 2 the intensity ratio becomes less temperature dependent than before. Curve 3 is a mean curve obtained for $n_{1 \mathrm{~s}}=10^{12} \ldots 10^{14} \mathrm{~cm}^{-3}$ with $T_{h}=T_{e}$. For $n_{1 \mathrm{~s}}<10^{12} \mathrm{~cm}^{-3}$, curve 3 approaches curve 2 with decreasing number density $n_{1 \mathrm{~s}}$. For details see Ref. 61 .

When one allows additionally for bound-bound electronic collisions between all levels and for bound-free electronic collisions the intensity ratio changes drastically. For a sufficiently high density ratio $n_{e} / n_{1 \mathrm{~s}}$ (in with $T_{e}$ as a parameter. These curves are the results of refined calculations. ${ }^{66}$ For $n_{e} \geq 10^{14} \mathrm{~cm}^{-3}$ the intensity ratio is practically $n_{e}$ - and $T_{e}$-independent!

It is interesting to calculate the intensity ratio I(4713)/I(4921) for an equilibrium plasma. One obtains

$$
\frac{\mathrm{I}(4713)}{\mathrm{I}(4921)}=\frac{g_{4^{\mathrm{I}} \mathrm{S}}}{g_{4^{3} \mathrm{D}}} \frac{\mathrm{A}_{4713}}{\mathrm{~A}_{4921}} \frac{\lambda_{4921}}{\lambda_{4713}} \exp \left(-\frac{E_{4^{3} \mathrm{D}}-E_{4^{1} \mathrm{~S}}}{k T_{e}}\right) \cong 0.31 .
$$

It follows from this that for $n_{e} \geq 10^{14} \mathrm{~cm}^{-3}$ the free electrons bring the upper state populations into mutual equilibrium. This result was already obtained by Strunnikov $^{62}$ in 1966 and is in agreement with the criteria for the establishment of partial equilibrium of the upper state populations. $^{63}$

More recently, Schieber et al. ${ }^{64}$ have tried to save the initial concept of determining $T_{e}$ by chosing other pairs of spectral lines. Their studies gave no improvement. Due to this unsatisfactory situation relative to the interpretation of the ratio of spectral line intensities Schmid ${ }^{65}$ proposed to use for the $T_{e}$-determination directly absolute spectral line intensities which are proportional to the upper state populations, the latter varying nearly exponentially with temperature. The proposed simplified collisional-radiative model is shown in Fig. 4. The $3^{1} \mathrm{D}$ level is chosen an upper level because of its special property within the level system: there is no direct radiative coupling with the ground and the metastable states; at low electron densities coupling with higher excited levels is still negligible. Since helium molecular ions $\mathrm{He}_{2}^{+}$are formed by collisions between a ground and an excited state atom, this process has consequently been included (see also later). Denoting by $X_{i}$ the population densities of level $i$ one obtains for the quasi-homogeneous quasi-stationary state the following system of rate equations for the three first excited levels (the $a_{i}$ and $a_{i j}$ denotereaction frequencies symbolized in Fig. 4):

$$
\left.\begin{array}{rrrr}
a_{10} X_{0}- & \left(a_{3}+a_{4}+a_{8}\right) X_{1}+ & a_{2} X_{2} & =0 \\
a_{20} X_{0}+ & a_{3} X_{1}-\left(a_{2}+a_{5}+a_{9}\right) X_{2}+ & a_{6} X_{3}=0 \\
a_{30} X_{0}+ & a_{4} X_{1}+ & a_{5} X_{2}-\left(a_{6}+a_{7}\right) X_{3}=0
\end{array}\right\}
$$

practice $n_{e} / n_{1 \mathrm{~S}}>10^{-3}$ is sufficient) one now obtains curves 4 of Fig. 2. In a large temperature range the intensity is nearly temperature-independent but has become $n_{e}$ dependent. This effect is more clearly demonstrated in Fig. 3 where the intensity ratio is given as a function of $n_{e}$

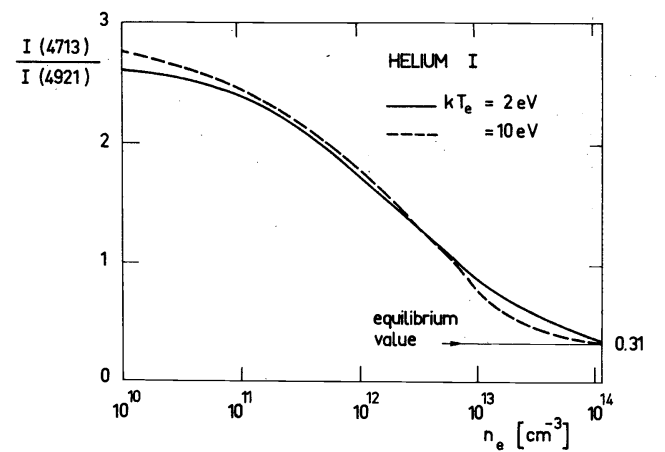

Fig. 3. Calculated intensity ratio $I(4713) / I(4921)$ after the collisional-radiative model; optically thin He plasma, after Drawin and Henning. ${ }^{61}$
Compared to this, the coronal model (only electronic excitation from ground state and radiative de-excitation towards level $2^{1} \mathrm{P}$ ) yield the equation

$$
a_{30} X_{0}-a_{6} X_{3}=0 \text {. }
$$

When the ground state density $X_{0}$ is known (for instance from a measurement of neutral gas temperature $T_{0}$ and total pressure $p, p \approx X_{0} k T_{0}$ ) the population densities can be calculated. Computed populations $X_{3}$ of the $3^{1} \mathrm{D}$ level are shown in Fig. 4 for an optically thick plasma. One clearly sees the effect of additional coupling between bound states, compared to the coronal model. It should be pointed out that reabsorption influences the results. Photon absorption in the $2^{1} \mathrm{P}-1^{1} \mathrm{~S}$ resonance transition increases the population of level $2^{1} \mathrm{P}$. Since the $2^{1} \mathrm{~S}$ level is strongly coupled to the $2^{1} \mathrm{P}$ level, the $2^{1} \mathrm{~S}$ population also increases. The increase of both the $2^{1} \mathrm{~S}$ and $2^{1} \mathrm{P}$ populations must lead to a non negligible increase of the $3^{1} \mathrm{D}$ population. Corresponding data may be found in a special report. ${ }^{66}$ Interpretation of measured population densities of opti- 


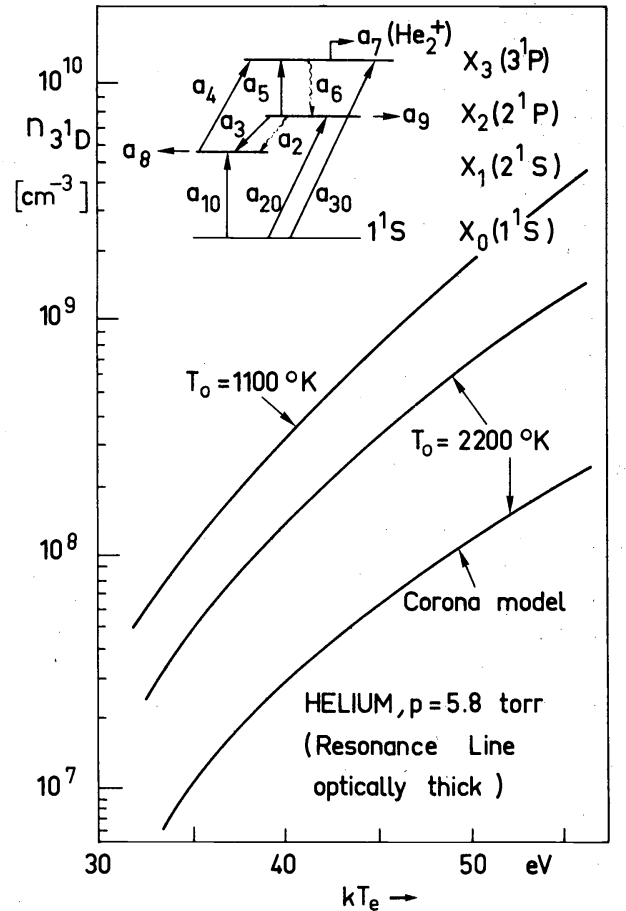

Fig. 4. Proposed simplified collisional-radiative model for spectroscopic determination of electron temperature from absolute line intensity measurement of a $\mathrm{He}$ line and calculated populations of $3^{1} \mathrm{D}$ level (after Schmid $\left.{ }^{65}\right)$. Read $\mathrm{X}_{3}\left(3^{1} \mathrm{D}\right)$ instead of $\mathrm{X}_{3}\left(3^{1} \mathrm{P}\right)$.

cally thick plasmas requires a profound study of the radiative transfer equation in connection with the rate equations for the particle densities.

New difficulties in the interpretation of the excited state populations arise at low electron densities $\left(n_{e}<10^{12} \mathrm{~cm}^{-3}\right)$ and high electron temperatures $\left(T_{e}>50,000^{\circ} \mathrm{K}\right)$ due to the possible existence of doubly excited helium atoms (so-called autoionizing states). The electronic excitation of the second bound electron and its de-excitation has an important influence on the populations of singly excited particles. At these plasma parameters the population densities of the singly excited particles are essentially determined by the excitation/de-excitation rates of doubly excited states. The few model calculations made until now give only a qualitative idea, and it is the author's feeling that definite quantitative results for the populations of helium levels at low electron densities and high electron temperature are still completely lacking.

It should be mentioned that astrophysicists face a similar situation when determining the helium abundances. Due to the much higher radiances they use the line pairs $\lambda=5876 \AA\left(3^{3} \mathrm{D}-2^{3} \mathrm{P}\right), \quad \lambda=6678 \AA$ $\left(3^{1} \mathrm{D}-2^{1} \mathrm{P}\right)$.

\section{Lithium-like ions}

In a series of papers Suckewer ${ }^{67-70}$ has calculated populations of a number of neutral and ionized species. A strong $n_{e}$-dependence of the ratio of spectral line intensities originating from alkaline-like levels with the same upper principal quantum number was found.

†Since particles of the Li-like sequence contain more than one bound electron, doubly excited particles may influence the populations of singly excited particles at high electron temperatures.
Consider for instance the Li-like sequence shown in Fig. 5 . When one assumes that the two levels $3^{2} \mathrm{D}$ and $3^{2} \mathrm{P}$ are only populated by electronic collisions from the ground level and depopulation is only due to spontaneous - de-excitation one would obtain a temperature-dependent line intensity ratio, as in the case of neutral helium. However, when one allows additionally for electronic collisions between all levels and also with the continuum the temperature dependence disappears. The line ratio becomes strongly $n_{e}$-dependent. This is shown in Fig. 6 for the CIV lines which have both been assumed to be optically thin. $\dagger$

\section{Li-like sequence: Li I, Be I, BII, C $\nabla, N \nabla, O Z$}

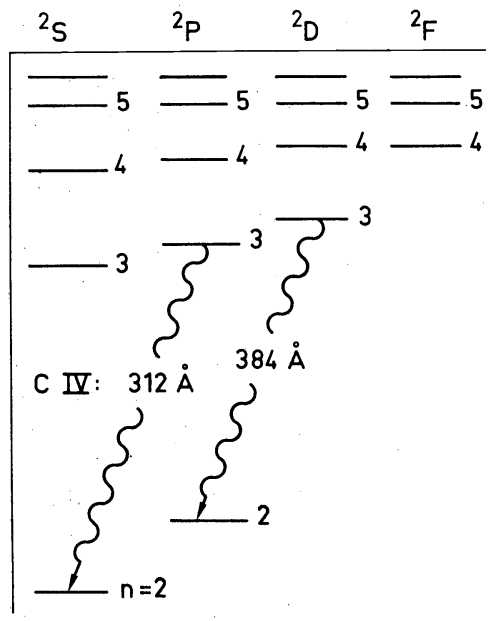

Fig. 5. Level system of Li-like sequence and line transitions considered by Suckewer. ${ }^{69}$

For sufficiently high ground state densities re-absorption occurs especially in the $\lambda=312 \AA$ line; but re-absorption can also be appreciable in the $\lambda=384 \AA$ line, since the $2^{2} \mathrm{~S}$ and $2^{2} \mathrm{P}$ states are strongly coupled by radiative and collisional effects. Before applying the curves for the optically thin case one has to check that re-absorption is indeed negligible.

Figure 7 shows calculated Boltzmann decrements $a_{i}$ for some levels of NV and O VI, also computed by Suckewer. ${ }^{68} a_{i}$ is defined as follows

$a_{\mathrm{i}}=$

$\left(n_{i} / n_{1}\right)$ calculated for non-L.T.E. plasma of given $n_{e}$ and $T_{e}$ $\left(n_{i} / n_{1}\right)$ Boltzmann for same $n_{e}$ and $T_{e}$

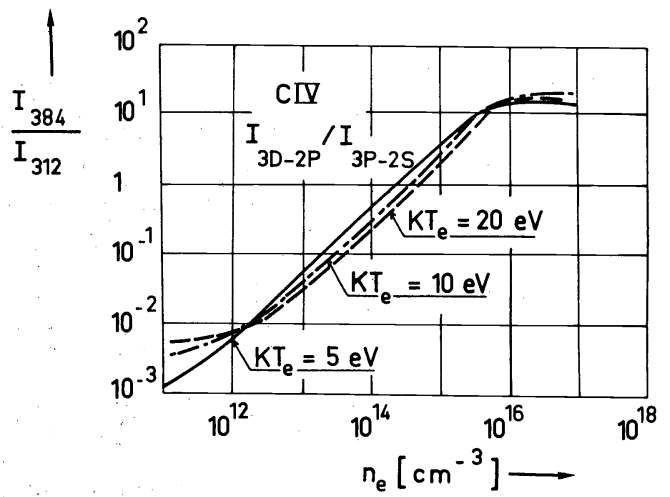

Fig. 6. Calculated intensity ratio $\mathrm{I}(384) / \mathrm{I}(312)$ for optically thin CIV lines (after Suckewer ${ }^{69}$ ). 


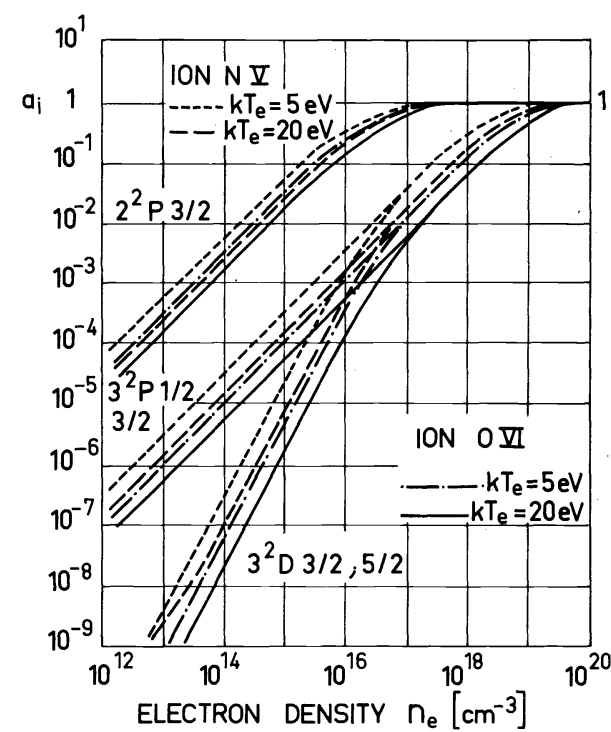

Fig. 7. Calculated Boltzmann decrement $a_{i}$ of the levels of $\mathrm{NV}$ and $\mathrm{O}$ VI, optically thin plasma (after Suckewer ${ }^{69}$ ).

$n_{1}$ is the ground state density. Since both excited state populations are proportional to $n_{1}, a_{i}$ becomes independent.from ground state density as long as the plasma is optically thin in the resonance lines. (For influence of radiative absorption on the $a_{i}$, see Drawin, $Z$. Naturforsch. 24a, 1492 (1969).)

\section{Influence of autoionizing electronic states}

The excitation of two (and eventually even more) electrons leads to doubly (multiply) excited states. In laboratory plasmas this will happen when particles containing more than one bound electron penetrate into a plasma containing energetic electrons, for instance by diffusion. Bound energy states lying energetically above the usual ionization limit are called autoionizing states, since the second excited electron can slip from a bound to a continuum state without making an inelastic (superelastic) transition. The existence of doubly excited states influences the populations of singly excited levels as a result of the following physical process:

(i) An energetic electron collides with an ion containing at least one bound electron. The free electron can excite the bound electron. During the excitation process the free electron just looses all its kinetic energy $E$. It can thus be captured by the ion. A doubly excited particle is formed; symbolically:

$$
\mathrm{A}^{+r}(h, l)+e\left(E, l^{\prime}\right) \rightarrow \mathrm{A}^{+(r-1)}\left(i^{\prime}, l^{\prime} ; j^{\prime \prime}, l^{\prime \prime}\right)
$$

$(h, i, j=$ principal quantum number, $1=$ azimuthal quantum number).

It is also possible that doubly excited states are formed by the direct excitation process

$$
\mathrm{A}^{+(r-1)}\left(1 l, 1 l^{\prime}\right)+e(E, l) \rightarrow \mathrm{A}^{+(r-1)}\left(i^{\prime}, l^{\prime} ; j^{\prime \prime}, l^{\prime \prime}\right) .
$$

(ii) Now, the one bound excited electron can return back into the continuum whereas the other one returns back to its initial state. This is the inverse process of

\footnotetext{
$\dagger \mathrm{di}=$ prefix for two (gr.)
}

reaction (21a) and is called autoionization. However, it is also possible that the doubly excited particle formed in reaction (21a) decays due to radiative de-excitation (and superelastic electronic collisions). This is dielectronic recombination. Thus, the following radiative decay scheme is possible:

$$
\mathrm{A}^{+(r-1)}\left(i^{\prime}, l^{\prime} ; j^{\prime \prime}, l^{\prime \prime}\right) \rightarrow\left[\begin{array}{ll}
\mathrm{A}^{+(r-1)}\left(i^{\prime}, l^{\prime} ; j^{\prime \prime}, l^{\prime \prime}\right)+e\left(E, l^{\prime}\right) & (21 \mathrm{~b}) \\
\underbrace{A^{+(r-1)}\left(i, l ; j^{\prime \prime}, l^{\prime \prime}\right)}_{\longrightarrow}+h \nu_{1} & (21 \mathrm{c})
\end{array}\right.
$$

In most cases, the electron configuration $(i, l)$ of the $\mathrm{A}^{+(r-1)}$ ion is that for the ground state, thus the particles $A^{+(r-1)}\left(i, l ; j^{\prime \prime}, l^{\prime \prime}\right), A^{+(r-1)}\left(i, l ; j^{\prime \prime \prime}, l^{\prime \prime \prime}\right)$ etc. represent singly excited species. Since the free electrons appearing in reaction (21a) are mostly captured in highly excited states (for usual radiative capture just the contrary holds) the latter become overpopulated compared to a plasma in which this capture process is omitted. The actual population densities depend in a complicated manner on the ensemble of all possible collision and radiation processes. The most important are:

$$
\begin{aligned}
& \mathrm{A}^{+(r-1)}\left(i, l ; j^{\prime \prime}, l^{\prime \prime}\right)+h \nu_{1} \rightarrow \mathrm{A}^{+(r-1)}\left(i^{\prime}, l^{\prime} ; j^{\prime \prime}, l^{\prime \prime}\right) \\
& \mathrm{A}^{+(r-1)}\left(i, l ; j^{\prime \prime}, l^{\prime \prime}\right)+e \rightleftarrows \mathrm{A}^{+(r-1)}\left(i, l ; j^{\prime}, l^{\prime}\right)+e \\
& \mathrm{~A}^{+(r-1)}\left(i, l ; j^{\prime \prime}, l^{\prime \prime}\right)+e \rightleftarrows \mathrm{A}^{+r}(i, l)+e+e \\
& \mathrm{~A}^{+(r-1)}\left(i, l ; j^{\prime \prime}, l^{\prime \prime}\right)+h \nu \rightleftarrows \mathrm{A}^{+(r-1)}\left(i, l ; j^{\prime \prime}, l^{\prime \prime}\right) \\
& \mathrm{A}^{+(r-1)}\left(i, l ; j^{\prime \prime}, l^{\prime \prime}\right)+h \nu \rightleftarrows \mathrm{A}^{+r}(i, l)+e .
\end{aligned}
$$

Reaction $\left(21 c^{\prime}\right)$ is the inverse of reaction (21c) and leads back to a doubly excited particle, the reactions (21d-21g) determine the relative distribution within the level system of the $A^{+(r-1)}$ particles.

Dielectronic recombination and its influence on excited state populations has been treated by many authors, the reader finds corresponding references in $(8,71,72)$. Figure 8 shows as an example the Saha decrements $b_{i}$ of neutral calcium without (broken curves) and with (solid curves) dielectronic recombination. $b_{i}$ is defined as follows:

$b_{i}=$

calculated non L.T.E. population $n_{\mathrm{i}}$ for given $n_{e}, T_{e}, \ldots$ Saha population $n_{i}$ Saha for same values $n_{e}, T_{e}, \ldots$

It should be pointed out that dielectronic recombination has an important effect on the ionization equilibrium at elevated electron temperatures and reduced pressures. Dielectronic processes play an important role in astrophysics and many laboratory experiments. Its influence decreases with decreasing temperature (process (21a) becomes inefficient) and increasing electron density (processes (21d) and (21e) dominate). This is the reason why it is generally so difficult to observe dielectronic recombination processes under laboratory conditions.

Dielectronic recombination has only been discovered $11 \mathrm{yr}$ ago by A. A. Burgess. It is characterized by the existence of two simultaneously excited electronic states $\dagger$ during monoelectron-ion recombination. One could im- 


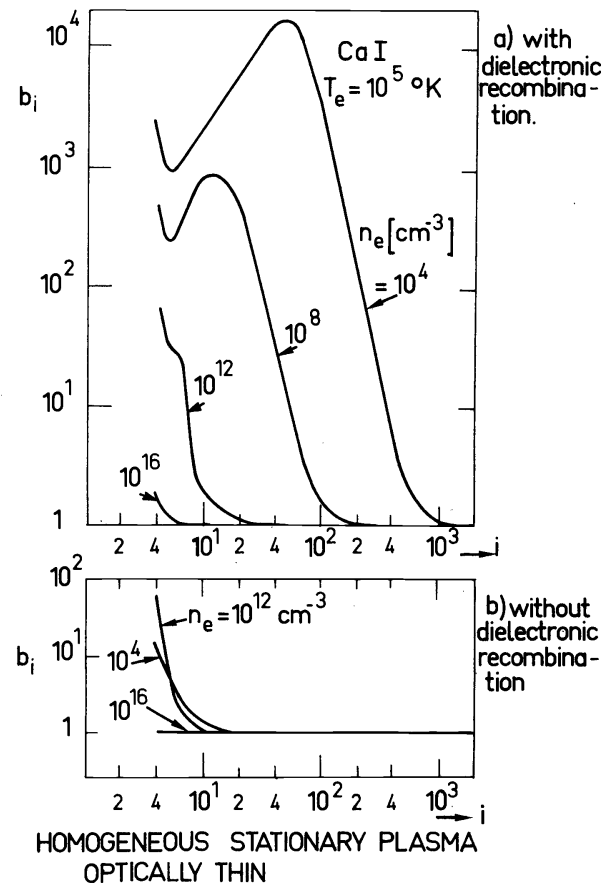

Fig. 8. Saha decrement $b_{i}$ of neutral calcium for optically thin homogeneous stationary plasma; a: with dielectronic recombination, after Burgess and Summers; $;^{72}$ b: without dielectronic recombination, the values have been calculated from the tables given $\mathrm{in}^{66}$ for helium and applying scaling for cross-sections.

agine a similar process in atom-molecule or moleculemolecule recombination:

Consider a free atom A colliding with radical BC. A can excite $\mathrm{BC}$ or break it up as an electron can in the collision $\mathrm{A}^{+r}(i, l)+e$. If, however, $\mathrm{A}$ approaches $\mathrm{BC}$ in suitable direction with appropriate energy and angular momentum it can excite a vibrational mode of the sub-system BC while being captured in another excitation mode of the whole system ABC. The efficiency of this capture process would depend on how strong the different modes are coupled. It can only work at very low densities.

\section{Influence of heavy particle collisions}

Until now we primarily considered the effect of different types of electronic collisions on the populations of electronically excited levels of atoms and atomic ions. These collisions prevail at sufficiently high temperatures and electron densities. However, at low degrees of ionization and sufficiently high gas pressure collisions between heavy particles (atoms and molecules) may considerably influence the populations of electronically and vibrationally excited states. In the special case of helium which is one of the most intensively studied gases because of its simple structure the following reactions can have a non-negligible effect on the populations:

(i) Collisional mixing of state populations according the reaction schemes

$$
\begin{aligned}
& \mathrm{He}(n, l)+\mathrm{He}\left(1^{1} S\right) \rightleftarrows \mathrm{He}\left(n, l^{\prime}\right)+\mathrm{He}\left(1^{1} S\right) \\
& \mathrm{He}(n, l)+\mathrm{He}\left(1^{1} \mathrm{~S}\right) \rightleftarrows \mathrm{He}\left(n^{\prime}, l^{\prime}\right)+\mathrm{He}\left(1^{1} \mathrm{~S}\right) .
\end{aligned}
$$

(ii) Ionizing collisions and three-body collisional recombination:

$$
\begin{aligned}
\mathrm{He}\left(n^{\prime}, l^{\prime}\right)+\mathrm{He}(n, l) \rightleftarrows \mathrm{He}^{+}+\mathrm{He}\left(1^{1} \mathrm{~S}\right)+e \\
\mathrm{He}\left(n^{\prime}, l^{\prime}\right)+\mathrm{He}_{2}(n, l) \rightleftarrows \mathrm{He}^{+}+e+\mathrm{He}\left(1^{1} \mathrm{~S}\right) .
\end{aligned}
$$

If $n^{\prime} \geq 2, n \geq 2$, we have collisional self-destruction through a kind of Penning ionization process; if $n^{\prime} \geq 1$, $n^{\prime}=1$ we obtain usual collisional ionization.

(iii) Associative ionization and dissociative recombination follow the scheme

$$
\mathrm{He}\left(n^{\prime}, l^{\prime}\right)+\mathrm{He}\left(1^{1} \mathrm{~S}\right) \rightleftarrows \mathrm{He}_{2}^{+}(v)+e .
$$

When $n^{\prime} \geq 3, l^{\prime}=n^{\prime}-1$ we get the usual Hornbeck-Molnar process which produces $\mathrm{He}_{2}{ }^{+}$ions in vibrational states $v$.

(iv) Under special conditions the following three-body collisional reactions may become important:

$$
\begin{aligned}
2 \mathrm{He}\left(2^{3} \mathrm{~S}\right)+\mathrm{He}\left(1^{1} \mathrm{~S}\right) & \rightleftarrows \mathrm{He}_{2}^{+}(v)+\mathrm{He}+e \\
2 \mathrm{He}\left(1^{1} \mathrm{~S}\right)+\mathrm{He}^{+} & \rightleftarrows \mathrm{He}_{2}^{+}+\mathrm{He} \\
\mathrm{He}_{2}^{+}(v)+2 e \rightleftarrows \mathrm{He}_{2}(n, v)+e & \stackrel{\mathrm{Le}_{2}{ }^{+}(v-1)+e .}{ }
\end{aligned}
$$

Channel (27b) is an auto-ionization process of an unstable electronically excited molecule in vibrational state $v$. This state is destroyed by ejection of an electron leaving the $\mathrm{He}_{2}{ }^{+}$ion in its electronic ground state and in vibrational state $v-1$. The rapid vibrational relaxation of $\mathrm{He}_{2}{ }^{+}$ions observed in afterglow plasmas may be due to this effect. The population distribution over quantum number $v$ has been calculated by Stevefelt. ${ }^{73}$

Quite similar reactions are possible for many other diatomic gases. For multi-atomic systems the possible reactions leading to a change of excited state populations are more numerous by far than for plasmas which contain only atoms and diatomic molecules and their ions.

Reactions of type (22a, b) may especially be of importance when the $\mathrm{He}\left(1^{1} \mathrm{~S}\right)$ atom is replaced by a molecule in vibrational state $v$, since translational and vibrational energies are now available for collisions which change the internal energy of the collision partners; symbolically:

$$
\begin{aligned}
& \mathrm{A}(n, l)+\mathrm{BC}(v) \rightleftarrows \mathrm{A}\left(n, l^{\prime}\right)+\mathrm{BC}(v) \\
& \mathrm{A}(n, l)+\mathrm{BC}(v) \rightleftarrows \mathrm{A}\left(n, l^{\prime}\right)+\mathrm{BC}\left(v^{\prime}\right) \\
& \mathrm{A}(n, l)+\mathrm{BC}(v) \rightleftarrows \mathrm{A}\left(n^{\prime}, l^{\prime}\right)+\mathrm{BC}\left(v^{\prime}\right) .
\end{aligned}
$$

Levels which are mainly populated (depopulated) by heavy particle collisions will show populations according to the gas rather than to the electron temperature. For such collisions to be effective (compared to electron collisions) the energy difference between the levels must be sufficiently small. This condition is fulfilled for fine structure levels within multiplet-terms and also for highly excited electronic states.

Our present knowledge about reactions between heavy particles, the potential energy curves, the energy dependence of the cross-sections and other atomic and molecular data is still scanty. The present situation does not permit detailed interpretation of measured data in terms of the mentioned reactions nor is it possible to calculate the non-thermal plasma state with a high degree of precision. There is however sufficient experimental evidence that all mentioned processes are important in plasmas of low degree of ionization. 
The influence of reactions (22a, b) and (23) on the level populations has been studied by Drawin et al ${ }^{74-77}$ and by Suckewer and Skorupski, ${ }^{70,78}$ reaction (24) together with reactions $(22 \mathrm{a}, \mathrm{b})(23)$ have been incorporated in model calculations by Schmid[65], whereas Stevefelt et al. ${ }^{73,79,80}$ and others primarily studied reactions (23b, 24-27a, b). The studies of Krysmanski ${ }^{81}$ refer to reactions of type $(22 \mathrm{a}, \mathrm{b})$ for the system $\mathrm{Fe}, \mathrm{Ar}$ and to reactions of type $(22 a, b)$ and $(28 b, c)$ for the system $\mathrm{Fe}, \mathrm{H}_{2} ; \mathrm{Fe}, \mathrm{N}_{2}$.

As an example and in order to demonstrate the importance of atom-atom collisions we show in Fig. 9 the percentage contribution of the Hornbeck-Molnar process to the depopulation rates of excited He levels. On the left-hand side, the percentage rates leading to population of quantum states $i$ are shown. The right-hand side of the same figure gives the percentage contributions to rates responsible for depopulating the same states. One clearly sees that for the special plasma parameters chosen the Hornbeck-Molnar process is the dominant depopulation mechanism at low and medium quantum numbers and it should be taken into account in the interpretation of measurements.

A second example is shown in Fig. 10 which represents a Boltzmann diagram for measured populations of $\mathrm{Fe}$ atoms for a 2 ampere iron arc operated in air at a pressure of 20 torr. Each dot corresponds to the populations of a multiplet-term as a function of excitation energy. The temperature determined from the Boltzmann slope depends on the couple of multiplets considered. One finds temperatures between $T=4300^{\circ} \mathrm{K}$ and $7000^{\circ} \mathrm{K}$. According to Krysmansky ${ }^{81}$ the lower of two temperatures is the gas temperature whereas the higher one should correspond to the electron temperature. I would like to emphasize that the explanation is probably valid as far as the relative values of the populations are concerned. The strong increase of the populations of terms of low excitation energies can also be explained by a diffusion effect (see next section).

The effect observed by Krysmanski has also been seen by Drawin et $a .^{76}$ on highly excited atoms.

Quite another process has been studied theoretically by Gudzenko et al. ${ }^{82}$ They compute possible population inversion with respect to the sodium ground state due to chemical reactions of type

$$
\begin{gathered}
\mathrm{Na}(n, l)+\mathrm{Cl}+\mathrm{M} \rightarrow \mathrm{NaCl}+\mathrm{M} \\
\mathrm{Na}(n, l)+\mathrm{F}+\mathrm{M} \rightarrow \mathrm{NaF}+\mathrm{M}
\end{gathered}
$$

where $M$ is an inactive third body. These authors showed that population inversion is indeed possible when the reaction rates for $(29 a, b)$ exceed a certain critical values.

From a thermodynamic point of view unexpected phenomena have been observed by many authors in the case of rare gas plasmas seeded with molecular gases, e.g. $\mathrm{He}+\mathrm{H}_{2}, \mathrm{He}+\mathrm{N}_{2}, \mathrm{Ar}+\mathrm{H}_{2}, \mathrm{Ar}+\mathrm{O}_{2}, \mathrm{Ar}+\mathrm{N}_{2}, \ldots$ Most of the observed features have not yet found satisfactory explanation. As an example we mention the measurements of Hefferlin ${ }^{83}$ who reports anomalous excitation of $\mathrm{N}_{2}{ }^{+}$in, a $\mathrm{Mn}\left(\mathrm{NO}_{3}\right)_{2}$ seeded argon plasma. The $\mathrm{N}_{2}{ }^{+}$first negative bands were enhanced ten order of magnitude over what thermodynamic equilibrium calculations predict.

\section{POPULATIONS OF EXCITED STATES IN INHOMOGENEOUS QUASI-STATIONARY PLASMAS WITH MAXWELLIAN ELECTRON VELOCITY DISTRIBUTION}

When a plasma is forced to occupy a limited volume it becomes more or less inhomogeneous. Inhomogeneities are accompanied by density and temperature gradients. These gradients lead to diffusion fluxes and, thus, to a change of the thermodynamic properties compared to the homogeneous state.

The diffusion terms appear on the left-hand side of the rate eqn $(4,5,7,8,9,12,13,15)$. These fluxes carry particles, photon momentum, and energy. The calculation of these fluxes is a difficult task, they depend on atomic and molecular properties, but also on the thermodynamic state. It is not the place here to discuss the problems in detail, however it appears to be useful to indicate at least the structure of the equations for a cylindrical geometry.

In the stationary state all terms of type $\partial\{\div\} / \partial t$ are zero. Denoting by $r$ the radial distance from the axis we
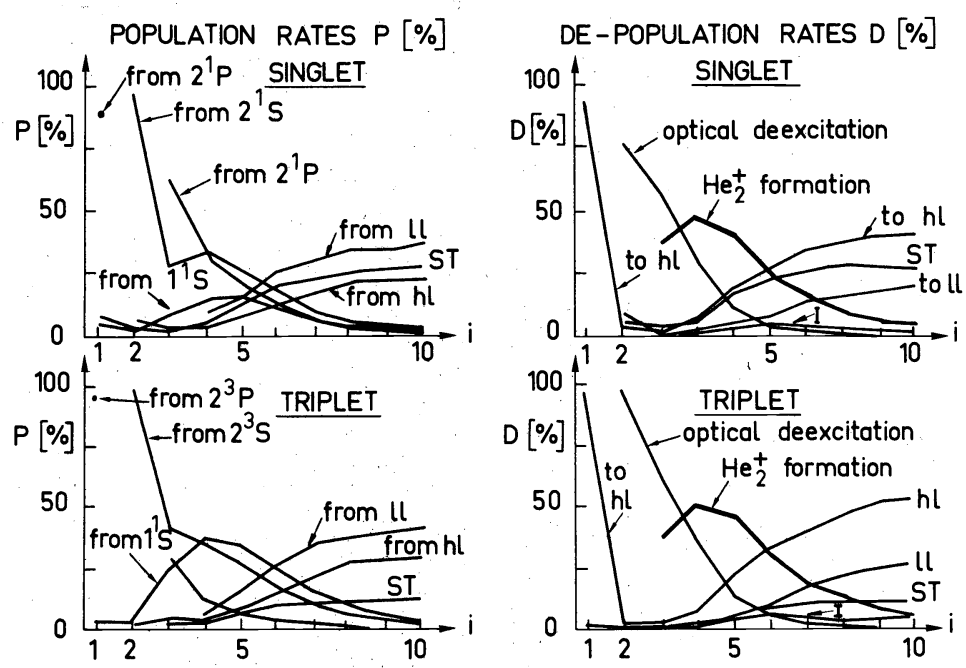

Fig. 9. Percentage contributions to rates leading to population (P) (left-hand side) and depopulation (D) (right-hand side) of the singlet and triplet states (after Schmid ${ }^{65}$ ). Total pressure $p=5.8$ torr. $n_{e}=3.8 \cdot 10^{11} \mathrm{~cm}^{-3}, T_{e}=3.5 \cdot 10^{4} \mathrm{~K}$, $T_{\text {gas }}=2.2 \cdot 10^{3} \mathrm{~K}$. The abbreviations have the following meaning: optical de-excitation $=$ total spontaneous de-excitation rate; $\mathrm{ST}=$ singlet $\rightarrow$ triplet collision transitions induced by $\mathrm{He}$ ground state atoms; $11=$ all lower levels: $h l=$ all higher levels; $i=$ principal quantum number if $i \geq 3, i=2$ is the $2 \mathrm{P}$ level, $i=1$ is the $2 \mathrm{~S}$ level. 


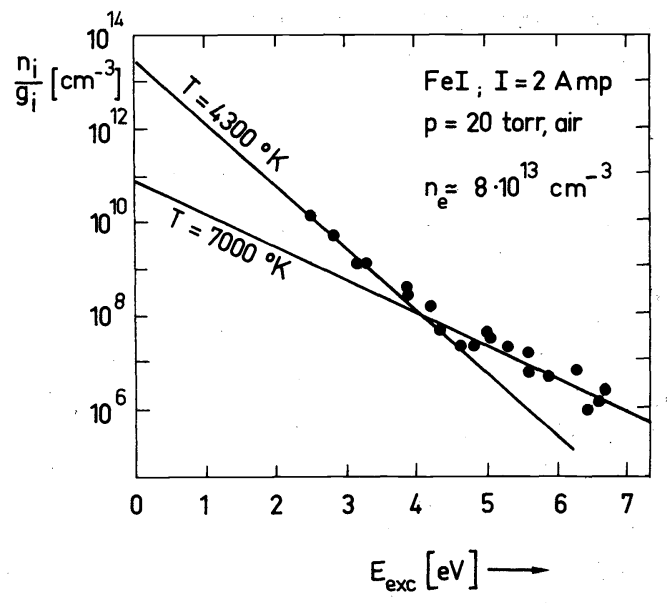

Fig. 10. Measured population densities $n_{i} / g_{i}$ of $\mathrm{Fe} I$ levels as a function of excitation energy $E_{\text {exc }}$ (after Krysmanski ${ }^{\mathbf{1}}$ ).

obtain the following equations:

For the transfer rate of particles $\mathrm{n}_{\mathrm{k}, \mathrm{i}}$ (eqn (14))

$$
\begin{aligned}
\frac{1}{r} \frac{\mathrm{d}}{\mathrm{d} r}\left(r j_{k, i}\right)= & \sum\left\{\begin{array}{l}
\text { all rates populating } \\
\text { level } i
\end{array}\right\} \\
& -\sum\left\{\begin{array}{l}
\text { all rates depopulating } \\
\text { level } i
\end{array}\right\}
\end{aligned}
$$

where $j_{k, i}$ is the diffusion flux $n_{k, i}\left\langle w_{k, i}\right\rangle_{r}$ of species $k$ in level $i$ diffusing in radial direction. It represents the difference of the number densities of particles crossing unit area in opposite direction. Classical considerations yield for instance the simple formula (with thermodiffusion neglected and $\bar{v}_{t h}=$ mean thermal velocity):

$$
j_{k, i}=-\frac{1}{3} \lambda_{k, i} \bar{v}_{t h} \nabla_{r} n_{k, i}=-\frac{1}{3} \lambda_{k, i} \bar{v}_{t h} \frac{\mathrm{d} n_{k, i}}{\mathrm{~d} r}
$$

where $\lambda_{k, i}$ is the mean free path of species $k$ in level $i$. Since $\lambda_{k, i}$ depends on the velocity-dependent crosssections and on the particle densities with which particles $k, i$ interact, $j_{k, i}$ cannot be calculated directly but follows together with solutions of the whole system of rate equations. For the electrons the rate equations have the same structure, one has to replace subscripts $k, i$ by subscript $e$ and to add a force term which accounts for the ambipolar field and eventual externally applied fields.

- For the transfer rate of momentum (eqn (6))

When magnetic fields and axial velocity gradients.are absent one can neglect the rate eqns (6) in a first approximation.

- For the transfer rate of energy (eqns $(7,8)$ )

\section{(i) Translational energy of electrons}

$$
\begin{aligned}
\frac{1}{r} \frac{\mathrm{d}}{\mathrm{d} r}\left(r q_{e}{ }^{t r}\right)-\sigma E^{2}= & \sum\left\{\begin{array}{l}
\text { net rate of } \\
\text { elastic collisions }
\end{array}\right\} \\
& +\sum\left\{\begin{array}{l}
\text { net rate of } \\
\text { inelastic collisions }
\end{array}\right\} \\
& +\sum\left\{\begin{array}{l}
\text { net rate } \\
\text { radiation }
\end{array}\right\}
\end{aligned}
$$

where $q_{e}^{t r}$ is the radial flux of translational energy of the electron gas. Since each electron transports on the average a kinetic energy of order $k T_{e}, q_{e}^{t r}$ is in a first approximation given by $k T_{e j} j_{e}$ where $j_{e}$ is the electron particle flux due to ambipolar and thermo-diffusion; further, $\sigma$ is the electrical conductivity; $E$ the electric field strength. Since the electrons lose (mainly) kinetic energy due to elastic collisions with the heavy particles (neutrals and ions) of temperature $T_{k}<T_{e}$, the first sum on the right-hand side of eqn (32) takes the form

$$
\sum\left\{\begin{array}{l}
\text { net rate of } \\
\text { elastic collisions }
\end{array}\right\}=-\sum_{k, i} \frac{4 m_{e}}{m_{k}}\left(k T_{e}-k T_{k}\right) \nu_{e}^{k, i}
$$

where $\nu_{e}^{k, i}$ is the elastic collision frequency between electrons and heavy particles. Finally, the electron gas can lose and gain kinetic energy due to inelastic and superelastic collisions respectively and due to radiation (e.g. bremsstrahlung) represented by the last two sums on the right-hand side of eqn (32).

(ii) Translational energy of heavy particles

$$
\left.\begin{array}{rl}
\frac{1}{r} \frac{\mathrm{d}}{\mathrm{d} r}\left(r q_{k . i}^{t r}\right)=\sum\left\{\begin{array}{l}
\text { net rate of } \\
\text { elastic collisions }
\end{array}\right\} & \\
& +\sum\left\{\begin{array}{l}
\text { net rate of } \\
\text { inelastic collisions }
\end{array}\right.
\end{array}\right\} .
$$

The first term on the right-hand side corresponds to heating by elastic collisions mainly with the electrons, thus:

$$
\sum\left\{\begin{array}{c}
\text { net rate } \\
\text { elastic collisions }
\end{array}\right\}=+\frac{4 m_{e}}{m_{k}}\left(k T_{e}-k T_{k}\right) \nu_{e}^{k, i}
$$

The second sum accounts for elastic and superelastic collisions which can often be neglected. Summing over all species $k$ and levels $i$ one obtains the total radial heat flux for heavy particle translational energy:

$$
q_{\text {heavy }}^{\text {tr }}=\sum_{k, i} q_{k, i \text {. }}^{\text {tr }}
$$

(iii) Internal energy of heavy particles

$$
\begin{aligned}
\frac{1}{r} \frac{\mathrm{d}}{\mathrm{d} r}\left(r q_{k, i}^{\mathrm{int}}\right)= & \sum\left\{\begin{array}{c}
\text { net rate } \\
\text { inelastic collisions }
\end{array}\right\} \\
& +\sum\left\{\begin{array}{l}
\text { net rate } \\
\text { radiation }
\end{array}\right\}
\end{aligned}
$$

The flux of internal energy, $q_{k, i}^{\mathrm{int}}$, is given by $j_{k, i} E_{k, 1 i}$ where $E_{k, 1 i}$ is the internal energy of species $k$ in a level $i$.

Summing up eqns (32-36) for all species one obtains for the total energy balance

$$
\sigma E^{2}=\frac{1}{r} \frac{\mathrm{d}}{\mathrm{d} r}(r q)+\nabla \cdot \mathbf{J}_{\text {photon }}
$$

where $q$ is the total radial heat flux and $\mathbf{J}_{\text {photon }}$ the total radiation flux vector. The latter follows from the equations of radiative transfer.

In order to show the influence of diffusion on the particle densities I will give two examples.

\section{Influence of diffusion on excited state populations}

Consider a homogeneous stationary hydrogen plasma of given electron density $n_{e}$ and electron temperature $T_{e}$. All populations can easily be calculated from a coupled system of rate equations of type (4) when a Maxwellian 
velocity distribution is assumed for the interacting particles. We have only to put the left-hand side equal to zero and to solve the system of algebraic equations. The resulting solutions are the homogeneous stationary state solutions and are denoted by $n_{i}^{\text {HS }}$. Especially for the ground state number density we write $n_{1}{ }^{\mathrm{HS}}$. To this ground state density belong the excited state population densities $n_{i}^{\mathrm{HS}} / g_{i}$ which lie in Fig. 11 on the curve labelled " $n_{1}{ }^{\mathrm{HS}}$ ". $g_{i}$ is the statistical weight. In semi-logarithmic representation one obtains a straight line for the higher excited states. The slope $\tan \alpha$ of this line yields the electron temperature $T_{e}$ and corresponds to the initial temperature $T_{e}=9000^{\circ} \mathrm{K}$ for which the calculations have been performed. (We note that $1 / i^{2}$ is proportional to the ionization energy $E_{1}{ }^{\mathrm{H}} / i^{2}$ of state $i$.)

When a particle flow rate is added the excited state populations and also the degree of ionization will change, since the collision rates change. We assume now a flux of ground state atoms entering from outside the plasma volume considered. These particles will cool the plasma. We assume now some electron heating mechanism to be effective which avoids cooling. Thus, the initial electron temperature will stay constant.

We chose for the divergency of the particle flux such a value that the new ground state density $n_{1}$ becomes equal to $(1 / 10) n_{1}{ }^{\mathrm{HS}}, 10 n_{1}{ }^{\mathrm{HS}}, \ldots$ The influx of particles increases the ground state density in the plasma volume considered. Thus, the excitation and ionization rates are increased. We require now that also the electron density $n_{e}$ does not change compared to the initial value assumed for the homogeneous stationary plasma. This is only possible when an ambipolar diffusion flux is added to the rate equation which determines the electron production rate. Loss of electrons due to ambipolar diffusion just cancels

$$
\begin{array}{ll}
\text { HYDROGEN PLASMA } & n_{e}=10^{14} \mathrm{~cm}^{-3} \\
\text { optically thick }\left(\Lambda_{1 j}=0\right) & T_{e}=9000^{\circ} \mathrm{K}
\end{array}
$$

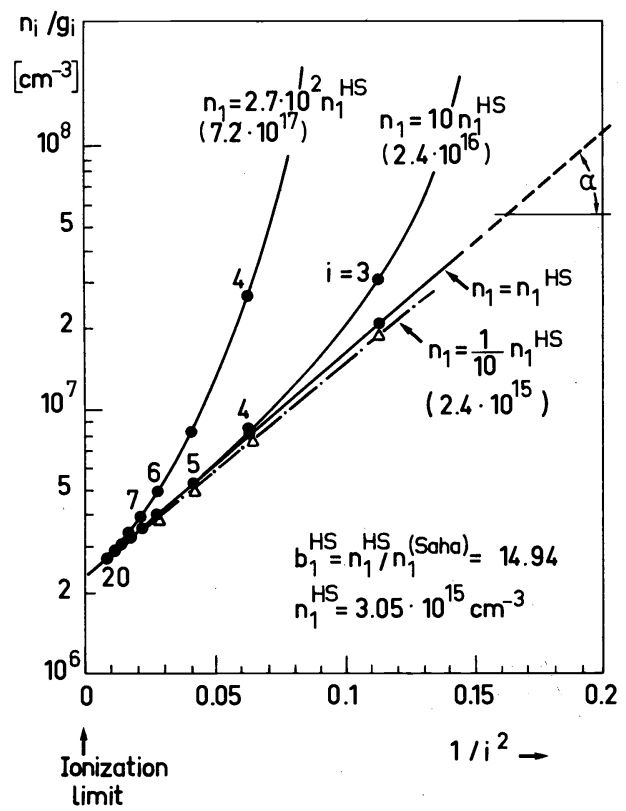

Fig. 11. Calculated population densities $n_{i} / g_{i}$ of atomic hydrogen in semi-logarithmic representation (Boltzmann plot). $i=$ principal quantum number, $\mathrm{HS}=$ homogeneous stationary state, $b_{1}{ }^{\mathrm{HS}}=$ Saha decrement for homogeneous stationary state. Resonance lines assumed to be optically thick. Further details see text. (After Drawin and Emard. ${ }^{95}$ ) the additional electron production rate due to the increased neutral particle density as a result of neutral particle diffusion. Thus, a new stationary state is obtained with same values of $T_{e}$ and $n_{e}$ as before. The system of equations which determines the excited state populations is now completely determined. We can compute the excited state populations as function of $T_{e}, n_{e}$ and the divergency of the diffusion flux $n_{1}\left\langle w_{1}\right\rangle$. The result of such calculations is shown in Fig. 11 by the curves labelled $n_{1}=(1 / 10) n_{1}{ }^{\mathrm{HS}}, n=10 n_{1}{ }^{\mathrm{HS}}, \ldots$ The curves above the one for a homogeneous stationary state plasma $\left(n_{1}{ }^{\mathrm{HS}}\right)$ are obtained when the particle flux yields an increase of the neutral particle density, the curve labelled $n_{1}=(1 / 10) n_{1}{ }^{\mathrm{HS}}$ is obtained when the diffusion of neutrals is directed in such a way that $n_{1}$ decreases compared to $n_{1}{ }^{\mathrm{HS}}$. The values of $\boldsymbol{\nabla} \cdot\left(n_{1}\left\langle\mathbf{w}_{1}\right\rangle\right)$ in units $\left(\mathrm{cm}^{-3} \mathrm{sec}\right)$ necessary to maintain the ground state density on their level $n_{1}$ have been added in parentheses. For the homogeneous stationary state, $\boldsymbol{\nabla} \cdot\left(n_{1}\left\langle\mathbf{w}_{1}\right\rangle\right)=0$ holds.

The populations now deviate from those for the homogeneous stationary state. The Boltzmann slope is not well defined. If one would apply the "Boltzmann slope method" for determining $T_{e}$, one would find a temperature which is too low in cases where diffusion increases the neutral particle density and too high temperature when diffusion yields a decrease of neutral particle density compared to the homogeneous stationary plasma. This effect has indeed been observed experimentally, see e.g. Refs. (84-93). The measurements of the population of iron levels by Krysmanski ${ }^{81}$ could also be explained by the diffusion effect.

For further details concerning model calculations with diffusion, see Refs. (94-100).

\section{Electronic state populations and temperature in a nitrogen plasma jet \\ Catherinot and $\mathrm{Sy}^{99}$ measured the population densities} of atomic nitrogen in a plasma jet (subsonic regime) and determined the electron temperature from the "Boltzmann slope". They found that the temperature depends on the quantum numbers of the levels considered. When introducing the diffusion terms in the equations for the level populations much higher temperatures are obtained, in agreement with theoretical arguments explained above. An off-axis temperature maximum will probably disappear when interactions with nitrogen atoms and molecules are taken into account. The heavy particle temperature calculated from the energy balance equation was found to be about three times lower than the electron temperature confirming that cold gas diffuses into the plasma and that translational equilibrium between electrons and heavy particles are not established. It is expected that the "vibrational temperature" also depends on the quantum numbers considered.

\section{POPULATIONS IN TRANSIENT QUASI-HOMOGENEOUS P̉LASMA WITH MAXWELLIAN VELOCITY DISTRIBUTION}

Transient plasmas are characterized by a more or less rapid variation of one or several plasma parameters. It is generally the electron or heavy particle temperature which changes first and which causes the variation of the densities. Transient phenomena occur in all types of pulsed discharges such as sparks, $\Theta$-pinches, waves carrying plasmas, shock waves and plasmas heated by high-frequency electro-magnetic radiation. The thermodynamic non-equilibrium state is described by the 
time-dependent rate equations for particle densities, momentum and energy. For very fast phenomena (heating by intense laser pulses of short duration) one has also to account for relaxation phenomena of the photon field. In connection with diagnostic methods a detailed knowledge of the transient behaviour of the various populations is absolutely necessary. The following problems shall briefly be discussed:

1. Temporal variation of electronic state populations after a sudden change of plasma parameters,

2. Influence of excited state populations on recombination and ionization,

3. Influence of heavy particle collisions on ionization and recombination.

\section{Transient behavior of the population of excited} electronic states.

To begin with, consider a transient homogeneous plasma. The particle densities are determined by eqn (4) which we write in the following form:

$$
\begin{aligned}
\frac{\partial n_{k, i}}{\partial t}=\left(\frac{\partial n_{k, i}}{\partial t}\right)_{\substack{\text { collision } \\
\text { radiation }}}=a_{i 1} n_{1}+a_{i 2} n_{2}+\cdots & +a_{i i} n_{i}+\cdots \\
& +a_{i p} n_{p}+\delta_{i} .
\end{aligned}
$$

The $a_{i j}(i \neq j)$ are interaction frequencies which lead to a population of level $i$ due to collisional-radiative interactions with all levels $j \neq i$. $a_{i i}$ represents the total interaction frequency leading to depopulation of level $i$, and $\delta_{i}$ is the total recombination rate into level $i$. On the right-hand side of eqn (38) we dropped the subscript $k$, for further details see e.g. Ref. (8). One has

$$
a_{i i}=-\left[\sum_{j \neq i}\left(a_{i j}+a_{j i}\right)+S_{i} n_{e}+\cdots\right]
$$

where $S_{i}$ is the ionization coefficients for level $i$. Any change of the plasma state is accompanied by a change of translational and internal energy, and also transfer of momentum may sometimes play an important role (e.g. compression in a $\theta$-pinch or choc wave). We shall neglect these phenomena.

When the system has reached the stationary state we have $\partial n_{k, i} / \partial t=0$ and eqn (38) takes the form

$$
a_{i i} n_{i}=-a_{i 1} n_{1}-a_{i 2} n_{2}-\cdots-a_{i p} n_{p}-\delta_{i}
$$

and permits the calculation of the stationary-state density $n_{i}$. Let us make the hypothesis that the populations $n_{i}(t)$ during the transient phase do not deviate too much from those for the stationary state. In this case the left-hand side of eqn (40) remains always of the same order of magnitude as the right-hand side, and eqn (38) can therefore be approximated

$$
\frac{\partial n_{k, i}}{\partial t} \cong a_{i i} n_{k, i}(t)=-n_{k, i}(t)\left(\sum_{j \neq i}\left(a_{i j}+a_{j i}\right)+n_{e} S_{i}\right) .
$$

The maximum error which one make is of the order of a factor two. The solution of eqn (41) is

$$
n_{k, i}(t)=n_{k, i}(0) \mathrm{e}^{a_{i i} t}=n_{k, i}(0) \mathrm{e}^{-t / \tau_{k, i}} \text {. }
$$

$\left\lceil n_{k, i}^{\mathrm{HS}}(\infty)\right.$ is the asymptotic value of the homogeneous stationary state population belonging to the new plasma parameters. In principle, this value is only reached at $t=\infty, n_{k, i}^{\mathrm{HS}}(\infty)$ is independent from time $t$.
Thus, a stationary state seems to be re-established for level $i$ after a time interval of order $\Delta t=\tau_{k, i}$ given by

$$
\tau_{k, i} \cong \frac{1}{\sum_{j \neq i}\left(a_{i j}+a_{j i}\right)+n_{e} S_{i}}
$$

$\tau_{k, i}$ represents the relaxation time of level $i$ to reach a stationary state relative to the other levels $j \neq i$ for the actual values of the plasma parameter $\left(n_{e}, T_{e}\right)$ after a sudden small variation of the latter. In eqn (43a) the main contribution comes very often from terms with $j=i-1$ and $j=i+1$. Then $\tau_{\mathrm{k}, \mathrm{i}}$ can be considered as the relaxation time of level i to come into a stationary state relative to the closest levels $\mathrm{i}+1$ and relative to the actual values of $\mathrm{n}_{\mathrm{e}}, \mathrm{T}_{\mathrm{e}}$. Without making any appreciable error one may still add in the denominator the term $\delta_{i} / n_{e}$ which accounts for direct recombination into level $i$. One thus obtains instead of eqn (43a) the relaxation time

$$
\tau_{k, i}=\frac{1}{\sum_{j \neq i}\left(a_{i j}+a_{j i}\right)+n_{e} S_{i}+\delta_{i} / n_{e}} .
$$

Quite similar relations are obtained for the relaxation times of vibrational and rotational states.

Numerical values for the relaxation times of electronically excited levels may be found in Refs. 8, 101, 102. Since the collision frequencies for the highly excited levels are large, one obtains extremely short relaxation times of the electronically excited levels of the order of $10^{-8}-10^{-14} \mathrm{sec}$.

An expression different from eqns $(43 \mathrm{a}, \mathrm{b})$ is obtained by the following considerations: for times $t \leq 0$ the stationary state population is $n_{k, i}(0)$. At time $t_{0}=0$ one suddenly changes the plasma condition which is followed by a temporal evolation of the populations towards a new stationary state. For times $t>0$ one may write

$$
n_{k, i}(t)=n_{k, i}(0)+\left(\frac{\partial n_{k, i}}{\partial t}\right)_{t=0} \mathrm{~d} t+\cdots
$$

where all higher-order times have been dropped. $\left(\partial n_{k, i} / \partial t\right)_{t=0}$ is the temporal variation of $n_{k, i}$ taken at time $t=0$ for the new plasma conditions. The new stationary state population is reached after a time interval $\Delta t \cong \tau_{k, i}$, $\tau_{k, i}$ being the relaxation time for establishing a new stationary state population relative to the new plasma parameters. Replacing in eqn (44) $n_{k, i}(t)$ by $n_{k, i}(\tau)$ and $d t$ by $\tau_{k, i}$ yields (for further details see Ref. 105.) $\dagger$

$$
\left.\tau_{k, i}=\left|\frac{n_{k, i}(\tau)-n_{k, i}(0)}{\left(\partial n_{k, i} / \partial t\right)_{t=0}}\right|=\mid \frac{n_{k, i}^{\mathrm{HS}}(\infty)-n_{k, i}(0)}{\left(\partial\left(n_{k, i}^{\mathrm{HS}}(\infty)-n_{k, i}\right) / \partial t\right)_{t=0}}\right] .
$$

When one puts $n_{k, i}(\tau)<n_{k, i}(0)$ and $\left(\partial n_{k, i} / \partial t\right)_{t=0}$ equal to eqn (41) for $t=0$ one obtains eqn (43a). However, in the general case eqn (45) will yield relaxation times which are different from those calculated from eqn (43a), especially in the case of extreme changes of the plasma parameters.

Level populations which are dominated by collisions follow the temporal evolution of the collision partners. This is for instance the case for highly excited electronic levels which are often dominated by electronic collisions. In this case the population densities of level $i$ are given by Saha's equation

$$
n_{k, i}=n_{k+1} n_{e} \frac{2 P_{k+1}(T)}{g_{k, i}} \frac{h^{3}}{\left(2 \pi m_{e} k T_{e}\right)^{3 / 2}} \exp \left(\frac{E_{k, i}-\Delta E_{k, k+1}}{k T_{e}}\right)
$$


where $g_{k, i}$ is the statistical weight of level $i, E_{k, i}$ the ionization energy of level $i$ and $n_{k+1}$ the particle density of ionic species $k+1$ resulting from an ionization of species $k$. When a change of the plasma conditions leads essentially to a change of the electron density the population densities will follow the variation of $n_{e}$. For a pure gas containing only neutrals and singly ionized particle one has $n_{k+1}=n_{e}$ and eqn (46) yields

$$
\frac{1}{n_{k, i}} \frac{\partial n_{k, i}}{\partial t}=\frac{2}{n_{e}} \frac{\partial n_{e}}{\partial t}
$$

i.e. the relaxation time of species in level $i$ for reaching a new stationary state is determined by the relaxation time for the electron density.

In order to obtain the "true relaxation times" (which account in a consistent manner for the simultaneous temporal evolution of all plasma parameters) one has to solve the whole system of coupled differential equations. A first step in this direction was done by Limbaugh and Mason ${ }^{103}$ and Cacciatore, Capitelli ${ }^{104,136}$ and Drawin ${ }^{105}$ who solved the system of time-dependent equations for $\mathrm{He}, \mathrm{H}$ and oxygen respectively. The numerical results depend on the special initial conditions chosen. It turns out that the relaxation times of highly excited levels calculated from the system of coupled equations are considerably larger than those obtained from eqn (42) for the same values of $n_{e}$ and $T_{e}$, especially for high lying levels.

As an example of these interesting calculations we show in Figs. 12a, b the temporal derivatives $\partial n_{i} / \partial t$ of excited hydrogen levels of principal quantum number $i$ in a pure hydrogen plasma in which reabsorption of photons is neglected. The initial conditions at time $t=0$ are $n_{e}(0)=1.10^{12} \mathrm{~cm}^{-3}, T_{e}(0)=16,000^{\circ} \mathrm{K}$.

The population of all excited levels at $t=0$ have been assumed to be populated according to Saha's equation. Figure $12 \mathrm{a}$ refers to a recombining plasma. It has been assumed that the ground level $i=1$ is at $t=0$ in equilibrium relative to $n_{e}=10^{12} \mathrm{~cm}^{-3}$ and $T_{e}=16,000^{\circ} \mathrm{K}$ (i.e. Saha decrement $b_{1}(t=0)=1$ ). Figure $12 \mathrm{~b}$ refers to an ionizing plasma and it has been assumed that the ground level $i=1$ is overpopulated relative to the solution for the homogeneous stationary state (Saha decrement $b_{1}(t=0=$ $\left.10^{6}\right)$. In both cases the plasma evolves towards the homogeneous stationary states solutions. This evolution can be seen in the figures.
The following general conclusions can be drawn from these model calculations: collisional-radiative coupling between (excited) states has a non-negligible influence on the temporal evolution of the thermodynamic state of a plasma. For times larger than $10^{-7}-10^{-6} \mathrm{sec}$ the temporal derivatives $\partial n_{k, i} / \partial t$, for the excited state populations are so small compared to $\partial n_{e} / \partial t$ for the electrons and $\partial n_{k, 1} / \partial t$ for the ground state atoms (ions) that all $\partial n_{k, i} / \partial t$ for the excited levels $(i>1)$ can be put equal to zero. (Exceptions occur when excited states lie close to the ground level; in this case the time derivatives of these levels must be taken into account.) This model is the so-called quasi-steady state model first introduced by Bates et al. ${ }^{106}$ It is simply characterized by the equations:

$$
\begin{aligned}
& \frac{\partial n_{e}}{\partial t}=-n_{+} n_{e} \alpha+n_{1} n_{e} S \\
& -\frac{\partial n_{e}}{\partial t}=\frac{\partial n_{1}}{\partial t}=a_{11} n_{1}+a_{12} n_{2}+\cdots+a_{1 i} n_{i}+\cdots \\
& +a_{1 p} n_{p}+\delta_{1} \\
& 0=a_{i 1} n_{1}+a_{i 2} n_{2}+\cdots+a_{i i} n_{i}+\cdots+a_{i p} n_{p}+\delta_{i} \\
& i>1
\end{aligned}
$$

to which similar equations for momentum and energy transfer have to be added. $\alpha$ and $S$ are the total collisional-radiation recombination and ionization coefficients. Equations (48c) represent a coupled system of algebraic equations for the populations $n_{i}>1$ as a function of $n_{e}, T_{e}$ and $n_{1}$. When these solutions are put into eqn (48b) one obtains $\partial n_{1} / \partial t$ as a function of $n_{e}, T_{e}$ and $n_{1}$. Since $\partial n_{1} / \partial t=-\partial n_{e} / \partial t$ one can determine $\alpha$ and $S$ by comparison of the coefficients of eqns (48a) and (48b).

\section{Influence of the number of excited levels on the recombination and ionization coefficients}

Electron density variations are considerably influenced by the excited state populations. For practical application of the quasi-steady state model it is important to know at what level $p$ the system (48c) can be truncated. Corresponding model calculations have been performed by Drawin and Emard ${ }^{107}$ and Cacciatore and Capitelli. ${ }^{108}$ Figure 13 shows the result of such calculations for the electron-ion recombination coefficient $\alpha$. One clearly sees

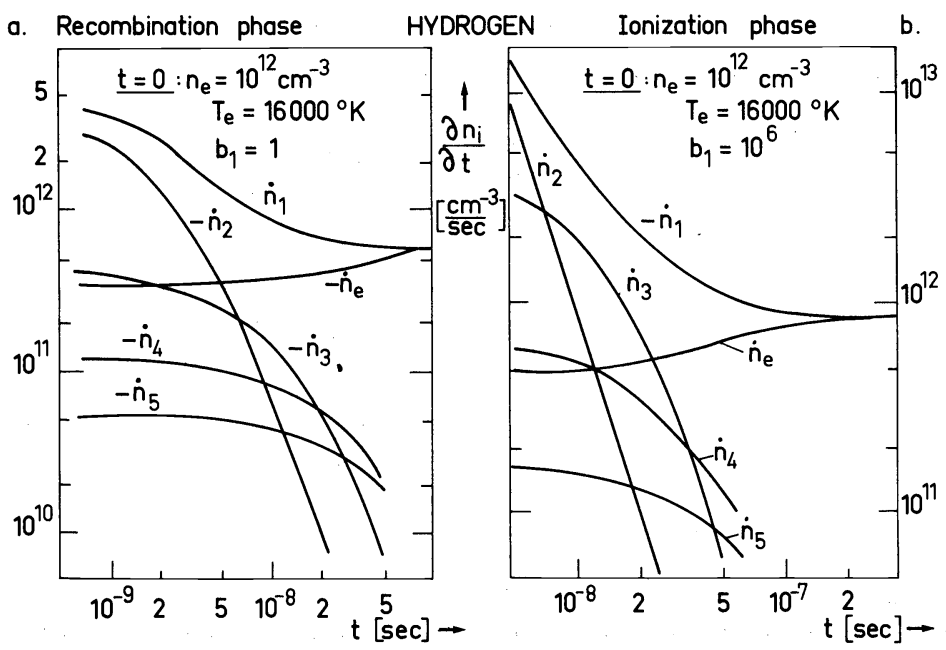

Fig. 12. Temporal evolution of particle densities in transient plasmas, after Cacciatore and Capitelli. ${ }^{104}$ a: recombining plasma, b: ionizing plasma. All lines and continua have been assumed to be optically thin. 


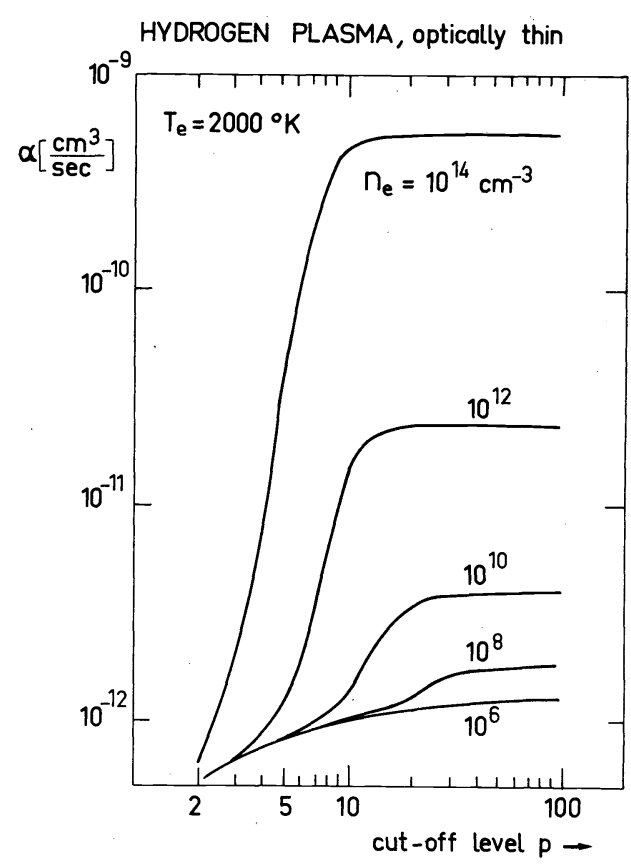

Fig. 13. Collisional-radiative recombination coefficient $\alpha$ of an optically thin hydrogen plasma, as a function of cut-off level $p$ which truncates the number of coupled equations. (After Drawin and Emard. ${ }^{107}$ )

the influence of the excited atomic levels on the recombination coefficient. According to these calculations one should take into account levels up to principal quantum number $p \cong 10-15$. Further examples are given in the mentioned papers and in 109,110 . There exist many other theoretical concepts for treating electron-ion recombination. A comparative study of seven different theoretical models has been undertaken by Drawin and Emard. ${ }^{111}$

It should be emphasized that electron-atomic ion recombination is the dominant recombination process in completely dissociated gases at sufficiently high degree of ionization. During the very early ionization phase or the late afterglow of a molecular gas, heavy particle collisions will have a non negligible influence on the plasma parameters. When this occurs the possible electronic, vibrational and rotational levels of the molecular system formed must be considered.

It should further be pointed out that the temporal behavior of a plasma depends in a high degree on the kind in which energy in gained or lost. A plasma in a constant heat bath will not change its composition until the temperature changes. Such a change can be imposed by changing the wall temperature. The change of plasma composition follows with a time constant which depends on particle and energy fluxes. The problem is discussed in detail in Refs. $(8,112-118)$.

\section{Influence of heavy particle collisions on recombination} and ionization

Heavy particle collision can have a considerable influence on the thermodynamic properties of a plasma. They become in any case important at low degree of ionization. Due to the many new energy states to be considered in heavy particle collisions model calculations become extremely complicated whereas large error can be committed in the interpretation of measurements when heavy particle collisions are not being accounted for. Figure 14 shows as an example calculated collisional recombination coefficients of a hydrogen plasma when reactions of type (22a, 22b and 23a) are added to electronic collisions and when a special type of electron collisions is assumed to be inefficient. The figure also shows the influence of photoabsorption in the free-bound continua. As the population densities and, thus, $\alpha$ and $S$ depend now additionally on the number density $n_{1}$ of hydrogen ground state atoms, the gas temperature intervenes in the evaluation of the reaction rates. For reasons of simplicity the latter has been put equal to $T_{e}$ in the calculations of Fig. 14. At low density $n_{1}, \alpha$ becomes constant. The absolute value does not only depend on the temperature but also on the special reaction processes considered. Omitting a collision process like $\mathrm{H}(i)+$ $e \rightleftarrows \mathrm{H}(\mathrm{j})+e$ has a much larger influence on the results than neglecting radiative absorption (see figure). At high neutral particle density $n_{1}, \alpha$ depends in a complicated manner on $n_{1}$. In a certain temperature and density region the recombination coefficient has a pronounced minimum (which is still a function of the degree of absorption).

As mentioned above, the actual recombination coefficient can depend on many different types of heavy particle collisions. Of special importance are those which change the heavy particle composition without changing the electron density (see e.g. reactions (26, and $27 \mathrm{a}, \mathrm{b})$ ). Any interpretation of measurements should therefore be based on models in which corresponding elementary reactions are taken into account.

\section{INFLUENCE OF LASER RADIATION ON EXCITED STATE POPULATIONS AND ON RECOMBINATION COEFFICIENTS}

Since high-power lasers are available, laser radiation has been widely used for studying multi-photon ionization

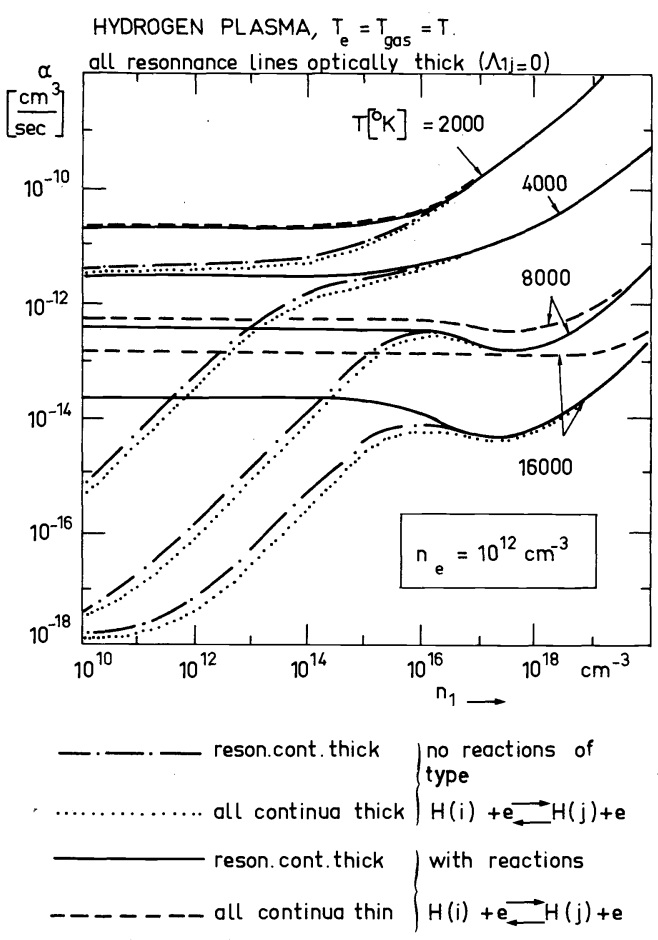

Fig. 14. Collisional-radiative recombination coefficient $\alpha$ of hydrogen plasma when heavy particle collisions are taken into account. Details see text. (After Drawin and Emard. ${ }^{107}$ ) 
processes in neutral gases, light scattering (Rayleigh, Raman and Thomson scattering) and plasma heating. In order to obtain measurable ionization breakdown of a gas and sufficiently intense scattering signals very powerful laser beams are needed. The power density lies generally above $10^{7} \mathrm{~W} / \mathrm{cm}^{2}$. The literature on laser-induced breakdown has recently been reviewed by Morgan ${ }^{119}$ and light scattering (especially Thomson scattering) has been described by Evans and Katzenstein. ${ }^{120}$

Much lower power densities are needed in experiments which consist in perturbing during a short time interval bound levels by radiative absorption of laser light tuned to specific bound-bound transitions. Relaxation measurements then permit the determination of atomic and molecular cross-sections or oscillator strengths for specific processes in which the perturbed levels intervene.

I would like to discuss here another effect, namely laser-induced photoionization and stimulated recombination in an ionized gas. Under certain conditions, these processes may have an important effect on the populations of excited electronic and molecular states. They may even be the dominant effect in an excited gas when the laser frequency $\nu_{L}$ is not in resonance with bound-bound transitions and if the power density is sufficiently high.

It is generally assumed (and verified by experiments) that photoionization (and also photodissociation) from excited states due to the plasma's own radiation field is an unimportant process under almost all laboratory conditions and that this effect need therefore not be taken into account in the interpretation of measurements. The situation changes drastically when a plasma is irradiated by an intense light beam, for instance a laser beam. We will briefly discuss the case of excited electronic states. First, single photon ionization can take place from all levels for which the photon energy $h \nu$ of the laser beam is equal to or larger than the ionization energy of these levels. Second, a sufficiently intense radiation field can also force the electrons to recombine with ions. This is radiation-stimulated recombination which must be added to the usual two-body collisional recombination. Thus, the following processes have to be considered.

$$
\mathrm{A}(i)+\left\{\begin{array}{l}
h \nu \rightleftarrows \mathrm{A}^{+}+e \\
2 h \nu \leftarrow \mathrm{A}^{+}+e+h \nu .
\end{array}\right.
$$

When the power densities of the laser radiation field are increased multi-photon ionization processes from excited levels may occur. For two-photon processes the following reaction scheme holds (for details see Ref. (137)):

$$
\begin{aligned}
& \mathrm{A}(i)+2 h \nu \rightleftarrows \mathrm{A}^{+}+e \\
& \mathrm{~A}(i)+3 h \nu \leftarrow \mathrm{A}^{+}+e+1 h \nu \\
& A(i)+4 h \nu \leftarrow \mathrm{A}^{+}+e+2 h \nu .
\end{aligned}
$$

For these processes to be efficient, $2 h \nu$ must be equal to or larger than the ionization energy of level $i$.

The photoionization processes lead to a lowering of the excited state populations whereas radiation-stimulated recombination increases the populations. The rate of the latter depends on the thermodynamic state of the electrons, i.e. on $n_{e}$ and $T_{e}$. Under certain conditions radiation-stimulated recombination can therefore play an important role, whereas it is negligible in other cases. Due to collisional-radiative coupling between all bound states the photoionization and stimulated recombination effects are also felt by states which do not suffer these effects directly. Consequently, the intensity of a more or less large number of spectral lines will be affected by the irradiation. Since electrons are re-ejected into the continuum by photoionization, the recombination coefficient also suffers a change during laser irradiation.

The influence of $\mathrm{CO}_{2}$ laser radiation on light emission of a helium afterglow plasma was first described by Kaplafka et al. ${ }^{121}$ and was explained as being due to light quenching originating from a photon-induced change of population of neutral or ionic helium molecular states. The same authors proposed in a later paper ${ }^{122}$ that this effect could be due to photoexcitation or photoionization of excited helium atoms. Chapelle et al. ${ }^{123}$ observed a decrease of spectral line intensities emitted from a stationary glow discharge in helium when irradiated by a c.w. $\mathrm{CO}_{2}$ laser beam (power density approx. $10^{5} \mathrm{~W} / \mathrm{cm}^{2}$ ). Further experimental results and the full theory for one-photon processes and for different types of plasmas and for different laser powers and laser frequencies have been given by Drawin et al. ${ }^{124}$ The same model has been used for calculations of the change of collisional-radiative recombination and ionization coefficients. ${ }^{125}$ Two-photon processes have most recently been incorporated in the model calculations by Emard. ${ }^{126}$ All these calculations are based on Maxwellian velocity distributions.

The broken curve in Fig. 15 shows the measured percentage lowering $\Delta n_{i} / n_{i}$ of the populations of excited helium triplet levels due to irradiation by a c.w. $\mathrm{CO}_{2}$ laser. $\Delta n_{i}$ denotes the difference $n_{i}^{\mathrm{WL}}-n_{i}$, where $n_{i}{ }^{\mathrm{WL}}$ is the population with laser irradiation, $n_{i}$ the one when the laser beam is cut-off. The solid curves are model calculations for two different electron densities close to the measured one. There is a general $10 \%$ lowering of all populations during laser irradiation as a result of gas heating. In addition to this one observes a level dependent decrease of populations which begins at principal

He glow discharge irradiated by $\mathrm{CO}_{2}$ laser

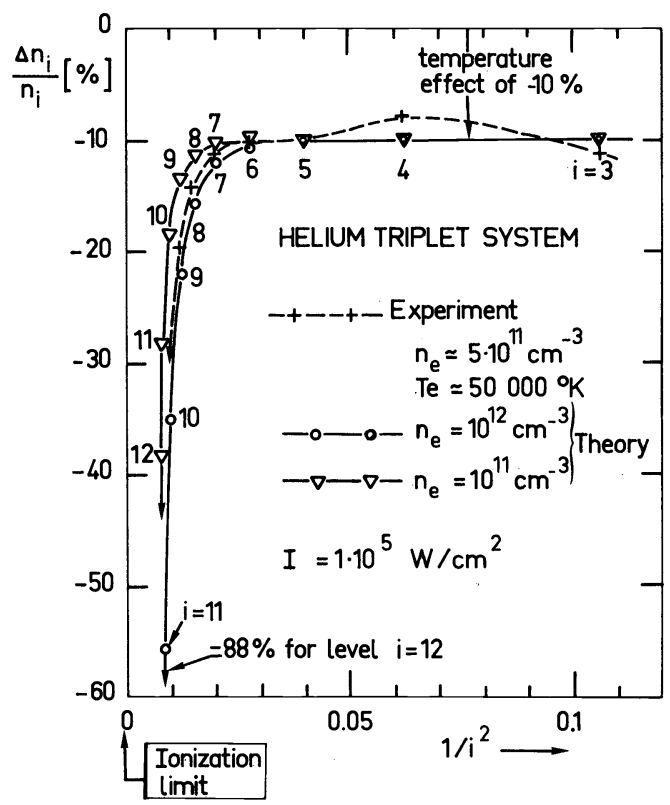

Fig. 15. Percentage lowering of helium excited state populations due to irradiation by $\mathrm{a} \mathrm{CO}_{2}$ laser beam. (After Chapelle et al. ${ }^{123}$ ) 
quantum number $i=6$. This lowering is due to the elementary reactions to which the excited states are exposed. Although the one-photon ionization process by $\mathrm{CO}_{2}$ laser photons $\left(\lambda_{L}=10.59 \mu, h \nu_{L}=0.117 \mathrm{eV}\right)$ in only effective for principal quantum numbers $i \geq 11$ the decrease of the corresponding populations is transmitted to the lower lying levels due to collisional-radiative coupling of all levels.

Figure 16 shows theoretical results of the relative lowering $n_{i}^{\mathrm{WL}} / n_{i}$ of populations of hydrogen atoms when a diffusion-dominated plasma of electron density $n_{e}=$ $10^{12} \mathrm{~cm}^{-3}$ and electron temperature $T_{e}=20,000^{\circ} \mathrm{K}$ is irradiated by a $\mathrm{CO}_{2}$ laser beam of different power densities I. Only one-photon processes have been taken into account in the model calculations. The broken curves are obtained when stimulated recombination is omitted, the solid curves when radiation-stimulated recombination is taken into account. One clearly sees that the latter fills up the bound states. Quite similar calculations have been performed for recombining plasmas. As an example we show in Fig. 17 the percentage lowering of the number of excited hydrogen atoms in a recombining plasma of electron temperature $T_{e}=1500^{\circ} \mathrm{K}$ irradiated by a $\mathrm{CO}_{2}$ laser beam of power density $3 \cdot 10^{5} \mathrm{~W} / \mathrm{cm}^{2}$. Due to the low electron temperature the excited levels are only populated from the continuum, excitation from the ground state $i=1$ is completely negligible. Therefore all levels "feel" the laser field although photoionization and stimulated recombination act only directly on level with $i \geq 11$. As before, the broken curves refer to calculations for which stimulated recombination was neglected.

At power densities of the order of $10^{5} \mathrm{~W} / \mathrm{cm}^{2}$ twophoton ionization processes from excited levels are still negligibly small. They begin to play a role when the power 'density exceeds $10^{6} \mathrm{~W} / \mathrm{cm}^{2}$. Figure 18 shows how the

Diffusion dominated

$\mathrm{H}$-Plasma irradiated by $\mathrm{CO}_{2}$ laser beam of different power densities I

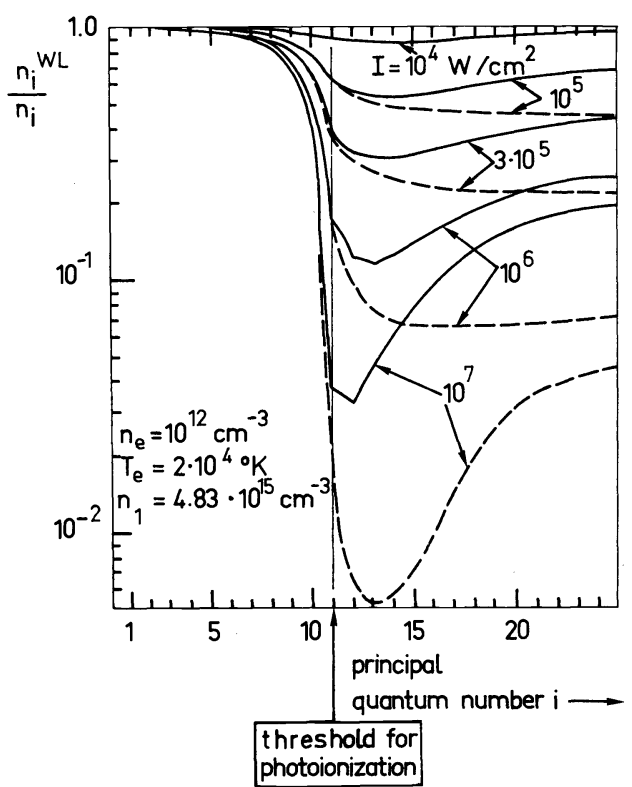

Fig. 16. Relative lowering of populations of excited hydrogen atoms in a diffusion-dominated plasma when irradiated by $\mathrm{a} \mathrm{CO}_{2}$ laser beam of different power densities. Broken curves: without stimulated recombination: solid curves: with stimulated recombination. (After Drawin et al. ${ }^{124}$ )
Recombining $\mathrm{H}$-plasma $\left(\mathrm{T}_{\mathrm{e}}=1500^{\circ} \mathrm{K}\right)$ irradiated by $\mathrm{CO}_{2}$ laser $\left(\mathrm{I}=3 \cdot 10^{5} \mathrm{~W} / \mathrm{cm}^{2}\right)$

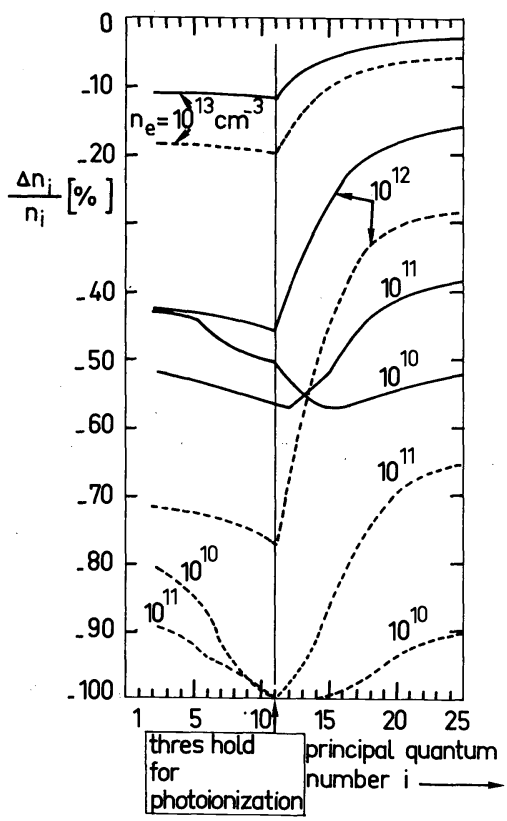

Fig. 17. Calculated percentage lowering of populations of excited hydrogen atoms in a recombining plasma when irradiated by $\mathrm{a} \mathrm{CO}_{2}$ laser beam of power density $\mathrm{I}=3 \cdot 10^{5} \mathrm{~W} / \mathrm{cm}^{2}$. Broken curves: without stimulated recombination; solid curves: with stimulated recombination. (After Drawin et al. ${ }^{124}$ )

$$
\begin{aligned}
& \text { Diffusion dominated H-Plasma }\left(n_{e}=10^{12} \mathrm{~cm}^{-3},\right. \\
& \left.n_{1}=4.83 \cdot 10^{15} \mathrm{~cm}^{-3}, T_{e}=20000^{\circ} \mathrm{K}\right) \\
& \text { irradiated by } \mathrm{CO}_{2} \text { laser }\left(I=10^{7} \mathrm{~W} / \mathrm{cm}^{2}\right)
\end{aligned}
$$

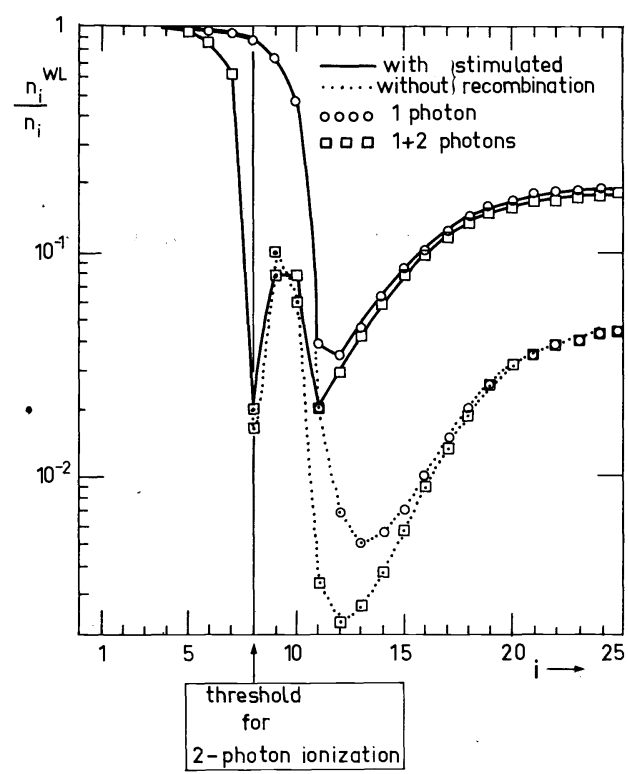

Fig. 18. Relative lowering of populations of excited hydrogen atoms in a diffusion dominated plasma when irradiated by a $\mathrm{CO}_{2}$ laser beam of power density $10^{7} \mathrm{~W} / \mathrm{cm}^{2}$. The figure shows the influence of one- and two-photon ionization processes. (After Emard. ${ }^{126}$ ) 
populations of a diffusion-dominated hydrogen plasma change when two-photon processes are included in the model calculations. Two $\mathrm{CO}_{2}$ laser photons together can ionize levels having principal quantum number $i \geq 8$. Due to the special $i$-dependence of the photoionization cross-sections each type of photoionization has its highest efficiency at and just above the threshold. The ionization efficiency decreases with increasing principal quantum number. This is the reason why the two-photon ionization process has only a small effect at high quantum number where the one-photon effect gives the main contribution to the lowering effect.

Laser irradiation can have a considerable effect on the temporal and spatial behavior of plasmas, since a change of the excited state populations also changes the collisional-radiative recombination and ionization coefficients (apart from eventual direct multi-photon ionization of ground state atoms not considered here; for hydrogen it becomes only effective for power densities larger than $10^{9} \mathrm{~W} / \mathrm{cm}^{2}$ ). Figure 19 shows how a $\mathrm{CO}_{2}$ laser beam can modify the collisional-radiative recombination coefficient of a recombining plasma at electron temperature $T_{e}=$ $1500^{\circ} \mathrm{K} . \alpha$ is the recombination coefficient without, $\alpha^{\mathrm{WL}}$ with laser irradiation. The results refer to a model in which only one-photon processes have been taken into account. The influence of two-photon processes on $\alpha^{\text {wl }}$ has been studied by Emard. ${ }^{126}$

Laser radiation does not only influence the electronic states, it also interacts with vibrationally and rotationally excited molecules. Due to the presence of vibrational and rotational states laser-induced excitation transfer through a series of molecular electronic states becomes possible. Such a two-step process in excited $\mathrm{H}_{2}$ has recently been observed by Dubreuil et al. ${ }^{127}$

\section{INFLUENCE OF INELASTIC AND SUPERELASTIC COLLISIONS ON VELOCITY DISTRIBUTION FUNCTIONS}

Any inelastic collision is accompanied by a loss of kinetic energy of the collision partners. This energy is regained in a superelastic collision. In thermal equilibrium there is an equal number of inelastic and superelastic collisions. On the average the velocity distributions

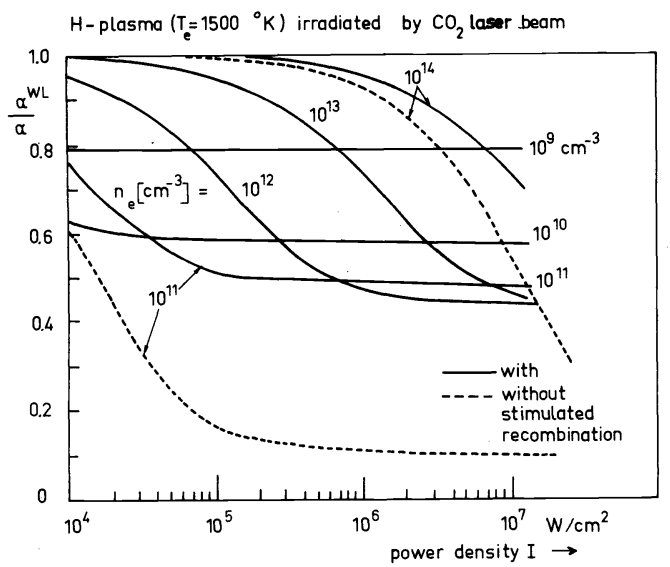

Fig. 19: Influence of $\mathrm{CO}_{2}$ laser radiation on the collisional-radiative recombination coefficient $\alpha$ of hydrogen plasma. $\alpha^{\mathrm{WL}}=$ with laser irradiation, $\alpha=$ without laser irradiation. (After Drawin and Emard. ${ }^{125}$ )

$\nmid$ More precisely, the number of collisions feeding the highenergy tail. remain Maxwellian. In non-equilibrium plasmas the populations do not necessarily obey a Boltzmann distribution due to some non equilibrated radiation or collision processes. It follows that an unequal number of inelastic and superelastic collisions result in a loss or gain of particles in special regions of the velocity distribution functions. The velocity distribution can also be brought into equilibrium by elastic collisions. If however, the number of such elastic collisions is small compared to the number of inelastic or superelastic collisions for the energy regions of interest the velocity distribution function can definitely deviate from a Maxwellian one. Deviation from a Maxwellian distribution function have a direct influence on the rate coefficients and, thus, on the populations and on the degree of ionization.

In plasmas of low degree of ionization the number of elastic Coulomb collisions between electrons is relatively small $\dagger$ compared to those making inelastic collisions during the ionization phase and also small compared to the number of superelastic collisions during the recombination phase. In the first case parts of the velocity distribution will be underpopulated compared to the equilibrium distribution, in the second case there will occur an overpopulation. Most sensitive is the highenergy tail where the number of redistributing collisions is smallest. The distribution function is also influenced by externally applied fields.

Calculations and measurements of many authors have shown (see Refs. 12-47) that serious deviations from a Maxwellian velocity distribution occur in plasmas of low degree of ionization. When these deviations are not taken into account large errors can be made when experimental results are interpreted.

As very often the main contributions to the excitation (ionization) and de-excitation (recombination) rates come from particles belonging to very different regions of the velocity distribution function it is sometimes useful to introduce two or more distribution functions of different densities and belonging to different temperatures. In this way it is possible to globally describe macroscopic features of a plasma without solving the Boltzmann equation. For details see Refs. 128-132. The existence of different velocity distribution functions for electrons and heavy particles and their effects on the populations of electronic, vibrational and rotational states is treated in Refs. 133-135.

Special problems arise in the boundary region between plasma and wall. Over distances of several mean free paths the distribution functions are non isotropic. A good approximation to the true distribution is the so-called bimodel function which is described by a superposition of two velocity distributions penetrating each other in opposite direction. ${ }^{138}$

\section{REFERENCES}

${ }^{1}$ D. H. Menzel (Ed.), Selected Papers on Physical Processes in Ionized Plasmas. Dower, New York (1962).

${ }^{2}$ A. R. Hochstim (Ed.), Kinetic Processes in Gases and Plasmas. Academic Press, New York (1969).

${ }^{3}$ J. W. Bond, K. M. Watson and J. A. Welch, Atomic Theory of Gas Dynamics. Addison-Wesley, Reading, Mass (1965).

${ }^{4}$ T. J. M. Boyd and J. J. Sanderson, Plasma Dynamics. Thomas Nelson \& Son, London (1969).

${ }^{5}$ D. C. Montgomery and D. A. Tidman, Plasma Kinetic Theory. McGraw-Hill, New York (1964).

${ }^{6}$ I. P. Shkarofsky, T. W. Johnston and M. P. Bachynski, The Particle Kinetics of Plasmas. Addison-Wesley, Reading, Mass (1966). 
${ }^{7}$ E. H. Holt and R. E. Haskel, Foundations of Plasma Dynamics. MacMillan, New York (1965).

${ }^{8} \mathrm{M}$. Venugopalan (Ed.), Reactions under Plasma Conditions. Vols. 1 and 2, Wiley-Interscience, New York (1971).

${ }^{9} \mathrm{M}$. Mitchner and Ch. H. Kruger, Partially Ionized Gases. WileyInterscience, New York (1973)

${ }^{10} \mathrm{G}$. Ecker, Theory of Fully Ionized Plasmas. Academic Press, New York (1972).

${ }^{11}$ D. H. Sampson, Radiative Contributions to Energy and Momentum Transport in a Gas. Wiley-Interscience, New York (1965).

${ }^{12}$ W. P. Allis, In: Handbuch der Physik. (edited by S. Flügge), Springer, Berlin (1956).

${ }^{13}$ M. N. Rosenbluth, W. M. MacDonnald and D. L. Judd, Phys. Review 107, 2nd series, 1 (1957): Fokker-Planck equation for inverse-square force.

${ }^{14}$ D. H. Sampson and J. Enoch, Electron distribution function and electrical conductivity of slightly ionized gas. Phys. Fluids 6, 28 (1963).

${ }^{15} \mathrm{~N}$. Peyraud, Theorie cinétique des plasmas, l'opérateur de collision inélastique dans le cas d'excitations et de désexcitations électroniques. J. Phys. 28, 147 (1967).

${ }^{16} \mathrm{~N}$. Peyraud, Action d'un champ électrique sur un gas faiblement ionisé-I. Influence des collisions inélastiques sur la "queue" de la fonction de distribution électronique: comportement asymptotique. J. Phys. 29, 201 (1968).

${ }^{17} \mathrm{~N}$. Peyraud, Action d'un champ électrique sur un gaz faiblement ionisé-II. Influence des collisions inélastiques sue la "queue" de la fonction de distribution électronique: Région du seuil d'ionization. J. Phys. 29, 747 (1968).

${ }^{18} \mathrm{~N}$. Peyraud, Action d'un champ électrique sur un gaz faiblement ionisé-III. Influence des collisions inélastiques sur la fonction de distribution électronique aux basses énergies. $J$. Phys. 29, 997 (1968).

${ }^{19}$ J. V. Dugan, F. A. Lyman and L. U. Albers, Solution of the Boltzmann and rate equations for the electron distribution function and state populations in non-equilibrium MHD plasmas, in: Electricity from MHD (edited by International Atomic Energy Agency, Vienna) Vol. 3, p. 85 (1966).

${ }^{20} \mathrm{~K}$. Woljaczek, Die Annäherung der Geschwindigkeitsverteilung der Elektronen an die Maxwell-Verteilung im Bereich der unelastischen Stösse. Beiträge aus der Plasmaphysik 5, 181 (1965).

${ }^{21} \mathrm{H}$. Rother, Geschwindigkeitsverteilung der Elektronen bei unelastischen Stössen. Z. Physik 173, 441 (1963).

${ }^{22} \mathrm{~K}$. Sauer and G. Wallis, Momentengleichungen mit Boltzmannund Fokker-Planck Stossintegralen. Beiträge aus der Plasmaphysik 5, 199 (1965)

${ }^{23}$ N. P. Carleton and L. R. Megill, Electron energy distribution in slightly ionized air under the influence of electric and magnetic fields. Phys. Rev. 126, 2089 (1962).

${ }^{24} \mathrm{E}$. A. Desloge, Velocity distribution of the electrons in an ionized gas in the presence of a homogeneous alternating electric field. Phys. Fluids 9, 982 (1966).

${ }^{25} \mathrm{~J}$. R. Viegas and C. H. Kruger, Electron distribution function for a nonequipartition plasma in a strong electric field. Phys. Fluids 12, 2050 (1969).

${ }^{26} \mathrm{~J}$. R. Viegas, Electron distribution function in a nonequipartition plasma. Report SU-IPR No. 139, Institute for Plasma Research, Stanford University, Stanford, California (1967).

${ }^{27}$ J. R. Viegas, Report NASA TN D-5548, Collision integrals for nonelastic processes in plasma kinetic theory. Ames Research Center, Moffett. Field, California (1969).

${ }^{28} \mathrm{~J}$. Oxenius, Effect of inelastic collisions on the tail of the electron velocity distribution. Z. Naturforsch. 25a, 101 (1970).

${ }^{29} \mathrm{~J}$. Oxenius, Optically thin hydrogen plasma with self-consistent electron velocity distribution. Z. Naturforsch. 25a, 1302 (1970).

${ }^{30} \mathrm{~J}$. Oxenius, Radiative transfer in the Lyman $\alpha$ spectral line with self-consistent electron velocity distribution. J. Quant. Spectr. Radiative Transfer 14, 731 (1974).

${ }^{31} \mathrm{~J}$. F. Shaw, M. Mitchner and C. H. Kruger, Effects of nonelastic collisions in partially ionized gases-I. Analytical solutions and results.Phys. Fluids 13, 325 (1970).
${ }^{32}$ J. F. Shaw, M. Mitchner and C. H. Kruger, Effects of nonelastic collisions in partially ionized gases-II. Numerical solution and results. Phys. Fluids 13, 339 (1970).

${ }^{33} \mathrm{~A}$. J. Postma, Calculated energy distribution functions and drift velocities in a Cesium discharge. Physica 43, 229 (1969).

${ }^{34} \mathrm{~A}$. J. Postma, Calculated electron energy distribution functions for discharges in helium and helium-argon mixtures. Physica 43, 581 (1969).

${ }^{35}$ A. J. Postma, Influence of several types of inelastic collisions on the electron energy distribution in helium. Physica 45, 609 (1970).

${ }^{36}$ R. J. Gould and R. K. Thakur, Deviation from a Maxwellian velocity distribution in low-density plasmas. Phys. Fluids 14, 1701 (1971).

${ }^{37} \mathrm{R}$. Winkler and J. Wilhelm, Geschwindikeitsverteilungsfunktion und Bilanzgrössen der Elektronen bei mittleren Ionisierungsgraden in Argon unter homogenen stationären Bedingungen. Beiträge aus der Plasmaphysik 11, 159 (1971).

${ }^{38} \mathrm{~W}$. L. Nighan, Electron energy distributions and collision rates in electrically excited $\mathrm{N}_{2}, \mathrm{CO}$ and $\mathrm{CO}_{2}$. Phys. Rev. A2, 1989 (1970).

${ }^{39}$ E. B. Sonin, Deviations from a Maxwellian electron energy distribution in a weakly ionized plasma. Sov. Phys. JETP 27 , 832 (1968).

${ }^{40} \mathrm{O}$. E. Pushkarev, Energy distribution of electrons and their concentration in a low temperature plasma. Sov. Phys. JETP 29, 305 (1969).

${ }^{41} \mathrm{E}$. Hantzsche, Zur Theorie der stationären Bewegung von Ionen im elektrischen Feld. Beiträge aus der Plasmaphysik 7, 277 (1967).

${ }^{42}$ W. E. Wells, P. Monchicourt, R. Deloche and J. Berlande, Theoretical computation of the effects of the metastable populations on electron energy balance and distribution function in a helium afterglow. Phys. Rev. A8, 381 (1973).

${ }^{43}$ H. Bender and K. G. Müller, Striations als Eigenlösungen der Elektronenbewegung im elektrischen Feld. Z. Physik 263, 299 (1973).

${ }^{44}$ H. G. Lergon and K. G. Müller, Örtliche Relaxation der Elektronen-Energieverteilung in hohen elektrischen Feldern. $Z$. Physik 268, 157 (1974).

${ }^{45} \mathrm{R}$. Winkler, Die Relaxation der Elektronengeschwindigkeitsverteilungsfunktion im homogenen feldfreien Lorentz Plasma. Ann. der Physik 31, 293 (1974).

${ }^{46} \mathrm{H}$. A. Classen, The influence of deviations from the Maxwellian electron distribution on the population densities in a low temperature monatomic plasma. Z. Naturforsch. 30a, 451 (1975).

${ }^{47} \mathrm{~S}$. Suckewer, Self consistent theory of population; ionization and electron distribution. Z. Physik 247, 354 (1971).

${ }^{48} \mathrm{~A}$. Ashkenas and F. S. Sherman, The structure and utilization of supersonic free jets in low density wind tunnels, in: Rarefied Gas Dynamics (edited by J. H. de Leeuw) Vol. 2, p. 84, Academic Press, New York (1966).

${ }^{49}$ R. J. Cattolica, An experimental study of translational non-equilibrium in free jet expansions. Report No. FM-72-6, College of Engineering, University of California, Berkeley (1972).

${ }^{50}$ Y.-S. Lou and R. E. Stickney, Velocity distribution measurements for near-free molecule flow through an orifice Phys. Fluids 13, 528 (1970).

${ }^{51}$ R. Cattolica, F. Robben and L. Talbot, Translational nonequilibrium in free jet expansion. Phys. Fluids 17, 1793 (1974).

${ }^{52} \mathrm{~F}$. S. Sherman, A source-flow model of viscous effects in hypersonic axisymmetric free jets. Arch. Mech. Stoss. 2, 471 (1964).

${ }^{53}$ B. B. Hamel and D. R. Willis, Kinetic theory of source flow exansion with application to the free jet. Phys. Fluids 9, 829 (1966).

${ }^{54}$ R. H. Edwards and H. K. Cheng, Distribution function and temperature in a monatomic gas under steady expansion into a vacuum in: Rarefied Gas Dynamics (edited by C. L. Brundin) Vol. 2, p. 819. Academic Press, New York (1967)

${ }^{55}$ N. C. Freeman, Solution of the Boltzmann equation for expanding flows. AIAA J. 5, 1696 (1967) 
${ }^{56} \mathrm{G}$. A. Bird, Breakdown of translational and rotational equilibrium in gaseous expansion. AIAA J. 8, 1998 (1970).

${ }^{57}$ R. L. Roy and Th. R. Govers, "Ideal" intensities of supersonic molecular beams. Can. J. Chem. 48, 1743 (1970).

${ }^{58} \mathrm{~W}$. Neumann, Spectroscopic methods of plasma diagnostics, in: Progress in Plasmas and Gas Electronics, (edited by R. Rompe and M. Steenbeck) Vol. 1. Akademie, Berlin (1975).

${ }^{59}$ S. P. Cunningham, U.S. Atomic Energy Commission Report WASH-289, pp. 279-287 (1975); see also C. B. Wharton, General Plasma Diagnostic Studies. Risö Report No. 18, Danish Atomic Energy Commission, Risö 1960.

${ }^{60}$ R. M. St. John, F. L. Miller and C. C. Lin, Absolute electron excitation cross sections of helium. Phys. Rev. 134, A888 (1964).

${ }^{61} \mathrm{H}$. W. Drawin and H. Henning, Über die Temperaturbestimmung aus dem Intensitätsverhältnis I(Triplett)/I(Singlett) zweier He I-Linien in nicht-thermischen Plasmen. Z. Naturforsch. 22a, 587 (1967).

${ }^{62} \mathrm{~V}$. M. Strunnikov, Limits of applicability of the method of measuring electron temperatures by the relative intensites of He I lines. Optics Spectroscopy 20, 1 (1966).

${ }^{63} \mathrm{H}$. W. Drawin, Validity conditions for local thermodynamic equilibrium, article in Vol. 1 of Ref. (8).

${ }^{64}$ D. Schieber, S. Gavril and M. S. Erlicki, Electron temperature determination in a low density helium plasma. Plasma Phys. 12 897 (1970).

${ }^{65} \mathrm{G}$. Schmid, Über das nichtthermische Gleichgewicht in einer Helium-Hohlkathodenentladung. Z. Naturforsch. 26a, 1901 (1971).

${ }^{66} \mathrm{H}$. W. Drawin and F. Emard, Tables of reduced population coefficients for the levels of atomic helium. Report EUR-CEAFC-697, Association Euratom-CEA, Fontenay-aux-Roses (1973). (See also report EUR-CEA-FC 534, Fontenay-aux Roses (1970).)

${ }^{67}$ S. Suckewer, Analysis of populations of excited states of ions and its application to electron temperature and density measurements. Plasma Phys. 10, 527 (1968).

${ }^{68} \mathrm{~S}$. Suckewer, Excitation and ionization of atoms and ions in a non-thermal plasma. J. Phys. B, 3, 380 (1970); 3, 390 (1970).

${ }^{69} \mathrm{~S}$. Suckewer, Populations of excited levels of atoms and ions: Electron temperature and density from relative line intensities of the ions CIV, NV and O VI. Phys. Rev. 170, 239 (1968).

${ }^{70}$ A. A. Skorupski and S. Suckewer, Non-thermal equilibrium parameters of weakly ionized plasmas. J. Phys. B, 7, 1401 (1974).

${ }^{71} \mathrm{E}$. Trefftz, Dielectronic recombination and competing processes, article in: Physics of the One-and Two-Electron Atoms (edited by F. Bopp and H. Kleinpoppen) North-Holland, Amsterdam (1969).

${ }^{72} \mathrm{~A}$. Burgess and $\mathrm{H}$. Summers. The effects of electron and radiation density on dielectronic recombination. Astrophys. $J$. 157, 1007 (1969).

${ }^{73}$ J. Stevefelt, Three-body capture-auto-ionization: A mechanism for vibrational de-excitation of molecular ions in a plasmas. Phys. Rev. A, 8, 2507 (1973).

${ }^{74} \mathrm{H}$. W. Drawin, Influence of atom-atom collisions on the population densities of excited atomic levels. Z. Naturforsch. 25a, 145 (1970).

${ }^{75} \mathrm{H}$. W. Drawin, Population densities and ionization equilibrium in two-temperature plasmas. Phys. Lett. 34A, 151 (1971).

${ }^{76} \mathrm{H}$. W. Drawin, F. Klan and H. Ringler, Investigation of excited states of helium in a stationary PIG-discharge. Z. Naturforsch. 26a, 186 (1971).

${ }^{77} \mathrm{H}$. W. Drawin and F. Emard, Atom-atom excitation and ionization in choc waves of the noble gases. Phys. Lett. 43A, 333 (1973).

${ }^{78} \mathrm{~S}$. Suckewer, Influence of heavy particle collisions on population and ionization equilibrium in weakly ionized plasmas. Phys. Lett. 32A, 507 (1970).

${ }^{79}$ J. Stevefelt, The decay of optically thick helium plasmas, taking into account ionizing collisions between metastable atoms or molecules. J. Phys. D, 4, 899 (1971).

${ }^{80} \mathrm{~J}$. Stevefelt and F. Robben, Spectroscopic study of the early afterglow of a recombining helium plasma. Phys. Rev. A, 5, 1502 (1972).

${ }^{81} \mathrm{~K}$. H. Krysmanski, Besetzung der Anregungszustände in
Bogenentladungen geringer Elektronendichte. Beiträge aus der Plasmaphysik 7, 349 (1967).

${ }^{82}$ L. I. Gudzenko, V. V. Evstigneev, S. S. Filippov and S. I. Yakovlenko, Recombination of a dense sodium plasma. High Temperature 12, 847 (1974).

${ }^{83} \mathrm{R}$. Hefferlin, Anomalous excitation of $\mathrm{N}_{2}{ }^{+}$in a seeded free plasma jet. J. Quant. Spectr. Radiative Transfer 12, 959 (1972).

${ }^{84} \mathrm{H}$. W. Drawin, M. Fumelli and G. Weste, Spectroscopic investigation of a hydrogen and helium plasma in a PIG discharge. Z. Naturforsch. 20a, 184 (1965).

${ }^{85}$ D. R. Simon and K. C. Rogers, Spectroscopic investigations of the helium-short arc. J. Appl. Phys. 37, 2255 (1966).

${ }^{86} \mathrm{~W}$. H. Venable and J. B. Shumaker, Observations of departures from equilibrium in a nitrogen arc. J. Quant. Spectr. Radiative Transfer 9, 1215 (1969).

${ }^{87} \mathrm{Ch}$. H. Kruger, Non-equilibrium in confined arc plasmas. Phys. Fluids 13, 1737 (1970).

${ }^{88} \mathrm{~J}$. Uhlenbusch, E. Fischer and J. Hackmann, Untersuchungen des Nichtgleichgewichtszustandes an stationären Edelgasplasmen unter Normaldruck. Z. Physik 239, 120 (1970).

${ }^{89} \mathrm{H}$. Boersch, J. Geiger and M. Topschowsky, Elektronenstossspektrometrische Bestimmung von Besetzungsdichten in einem Heliumplasma. Z. Naturforsch. 26a, 198 (1971).

${ }^{90}$ F. P. Incropera and E. S. Murrer, Spectroscopic measurements for atmospheric nitrogen and helium arcs. J. Quant. Spectr. Radiative Transfer 12, 1369 (1972).

${ }^{91}$ G. Pichler, V. Vujnovič, A. M. Tonejc and K. Acinger, A study of excitation conditions in a free-burning arc between $\mathrm{Cu}$ electrodes with currents from 1 to 15 A. Spectrochim. Acta 27B, 273 (1972).

${ }^{92}$ C. C. Hwang, Spectroscopic intensity measurements of atmospheric-pressure helium arc. J. Quant. Spectr. Radiative Transfer 12, 783 (1972).

${ }^{93}$ D. Garz, Nichtgleichgewichtseffekte im Argonkaskadenbogen im Duckbereich von 0,2 atm. bis 5,0 atm. Z. Naturforsch. 28a, 1459 (1973).

${ }^{94} \mathrm{H}$. W. Drawin, Besetzungsdichten angeregter Wasserstoffniveaus in stationären Plasmen unter Berücksichtigung der Diffusion von Neutralteilchen. Z. Physik 186, 99 (1965).

${ }^{95} \mathrm{H}$. W. Drawin and F. Emard, On the determination of electron temperature in diffusion-dominated non-L.T.E. plasmas. $Z$. Naturforsch. 28a, 1289 (1973).

${ }^{96} \mathrm{H}$. W. Drawin, F. Emard and K. Katsonis, Calculation of population densities of helium atoms in non-L.T.E. plasmas. $Z$. Naturforsch. 28a, 1422 (1973).

${ }^{97} \mathrm{R}$. T. Taussig, Theoretical model for non-equilibrium helium arc plasma. J. Quant. Spectr. Radiative Transfer 10, 449 (1970). ${ }^{98} \mathrm{~J}$. Uhlenbusch, Non-equilibrium effects in arc discharges, article in: Gaseous Electronics, Some Applications (edited by J. W. M. MacGowan and P. K. John) North-Holland, American Elsevier (1974).

${ }^{99} \mathrm{~A}$. Catherinot and A. Sy, Electron temperature determination from non-L.T.E. populations in a nitrogen plasma jet. $Z$. Naturforsch. 30a, 1143 (1975).

${ }^{100}$ T. L. Eddy, E. Pfender and E. R. G. Eckert, Spectroscopic mapping of the non-equilibrium between electron and excitation temperatures in a $1 \mathrm{~atm}$. helium arc. Plasma Sci. (I.E.E.E. Trans. P.S.) 1, 31 (1973).

${ }^{101}$ R. W. P. McWhirter and A. G. Hearn, A calculation of instantaneous population densities of the excited levels of hydrogen-like ions in a plasma. Proc. Phys. Soc. 82, 641 (1963).

${ }^{102} \mathrm{H}$. W. Drawin, Relaxation times for establishing steady-state population in optically thin and thick plasmas. J. Quant. Spectr. Radiative Transfer 10, 33 (1970).

${ }^{103} \mathrm{C}$. C. Limbaugh and A. A. Mason, Validity of the quasisteady state and collisional-radiative recombination for helium plasmas. I. Pure afterglows. Phys. Rev. A4, 2368 (1971).

${ }^{104} \mathrm{M}$. Cacciatore and M. Capitelli, Populations densities and ionization coefficients of fast transient hydrogen plasma. $Z$. Naturforsch. 30a, 48 (1975).

${ }^{105}$ M. Cacciatore, M. Capitelli and H. W. Drawin, Relaxation times for establishing quasi-stationary state populations in non-thermal plasmas. Physica 82C (1976). In press.

${ }^{106}$ D. R. Bates, A. E. Kingston and R. W. P. McWhirter, 
Recombination between electrons and atomic ions, I. Optically thin plasmas-II. Optically thick plasmas. Proc. Roy. Soc. A, 267, 297 (1962); A, 270, 155 (1962).

${ }^{107} \mathrm{H}$. W. Drawin and F. Emard, The role of particular collision and radiative processes in recombination. Z. Physik 270, 59 (1974).

${ }^{108} \mathrm{M}$. Cacciatore and M. Capitelli, Non L.T.E. populations and related quantities for $\mathrm{H}-\mathrm{H}^{+}-e$ plasmas as a function of the cut-off level. $Z$. Naturforsch. 29a, 1507 (1974).

${ }^{109} \mathrm{H}$. W. Drawin and F. Emard, Collisional-radiative volume recombination and ionization coefficients for quasi-stationary helium plasmas. Z. Physik 243, 326 (1971).

${ }^{110} \mathrm{H}$. W. Drawin and F. Emard, Collisional-radiative volume recombination and ionization coefficients for $\mathrm{He}^{+}-\mathrm{He}^{2+}-e$ plasmas. Z. Physik. 253, 100 (1972).

${ }^{11} \mathrm{H}$. W. Drawin and F. Emard, Comparative study of existing theories for volume ion-electron recombination in plasmas. Beiträge aus der Plasmaphysik 15, 273 (1975).

${ }^{112} \mathrm{D}$. R. Bates and A. E. Kingston, Recombination and energy balance in a decaying plasma; $\mathrm{I}$. $\mathrm{H}-\mathrm{H}^{+}-e$ plasma, $\mathrm{II}$. $\mathrm{He}-\mathrm{He}^{+}-e$ plasma. Proc. Roy. Soc. A, 279, 10 (1964); A, 279, 32 (1964).

${ }^{113}$ A. A. Newton and C. M. Sexton, The decay of highly ionized hydrogen and deuterium plasmas. J. Phys. B, 2, 1069 (1969).

${ }^{114}$ T. P. Sorensen and M. C. Sexton, Thermal conduction energy loss in an argon afterglow. J. Phys. D, 3, 1696 (1970).

${ }^{115} \mathrm{~J}$. C. Cronin and M. C. Sexton, Mechanisms of electron loss in a hydrogen afterglow. Proc. Roy. Irish Acad. 69, (A) 1 (1970).

${ }^{116} \mathrm{~B}$. V. Donovan and M. C. Sexton, Thermal conduction energy loss in a helium afterglow. J. Phys. D, 3, 1240 (1970).

${ }^{117}$ H. E. Wilhelm, Nonlinear Initial-boundary-value problem for convection, diffusion, ionization and recombination processes. J. Math. Phys. 13, 252 (1972).

${ }^{118} \mathrm{~J}$. Boulmer, C. Sol and J. C. Gauthier, Direct measurement of diffusion cooling in an afterglow plasma. Phys. Lett. 41A, 83 (1972).

${ }^{119}$ C. G. Morgan, Laser-induced break-down. Rep. Prog. Phys. 38, 621 (1975).

${ }^{120} \mathrm{D}$. E. Evans and J. Katzenstein, Laser light scattering in laboratory plasmas. Rep. Prog. Phys. 32, 207 (1969).

${ }^{121}$ J. P. Kaplafka, H. Merkelo and L. Goldstein, Quenching of helium afterglow by infrared photons. Appl. Phys. Lett. 15, 113 (1969).

${ }^{122}$ J. P. Kaplafka, H. Merkelo and L. Goldstein, Appl. Phys. Lett. 19, 197 (1971).

${ }^{123}$ J. Chapelle, B. Bubreuil, H. W. Drawin and F. Emard, Lowering of excited state populations of $\mathrm{He} \mathrm{I}$ in a He-plasma under the influence of laser radiation. Phys. Lett. 44A, 201 (1973).
${ }^{124}$ H. W. Drawin, F. Emard, B. Dubreuil and J. Chapelle, Lowering of the populations of excited hydrogen and helium atoms in plasmas irradiated by a laser beam. Beiträge aus der Plasmaphysik 14, 103 (1974).

${ }^{125} \mathrm{H}$. W. Drawin and F. Emard, Influence of laser radiation on the collisional-radiative recombination and ionization coefficients. Z. Physik 266, 257 (1974).

${ }^{126} \mathrm{~F}$. Emard, Influence of two-photon ionization processes on the populations of excited hydrogen atoms in non-L.T.E. plasmas. Physica 82C (1976). In press.

${ }^{127}$ B. Dubreuil, H. W. Dradin and J. Chapelle, Excitation transfer between excited electronic states of the $\mathrm{H}_{2}$ molecule due to interaction with $\mathrm{CCO}_{2}$ laser beam. Z. Physik B, 20, 117 (1975).

${ }^{128} \mathrm{H}$. W. Drawin, Anregung und Ionisation eines Wasserstoffplasmas unter dem Einfluss zweier Elektronengruppen unterschiedlicher Temperature und Dichte. Annalen d. Physik 17, 374 (1966).

${ }^{129} \mathrm{P}$. A. Büger und W. Scheuermann, Messungen von Anregungstemperaturen in der Hochstromhohlkathode.Z. Physik. 216, 248 (1968).

${ }^{130} \mathrm{~L}$. Vriens, Energy balance in low-pressure gas discharges. $J$. Appl. Phys. 44, 3980 (1973).

${ }^{131} \mathrm{~L}$. Vriens, Two and three-electron group models for lowpressure gas discharges. J. Appl. Phys. 45, 1191 (1974).

${ }^{132} \mathrm{H}$. W. Drawin, Ionization-recombination in a helium plasma containing two electron groups of different temperatures. $Z$. Naturforsch. 31a (1976). To be published.

${ }^{133} \mathrm{P}$. A. Büger, Spektroskopische Untersuchungen der Plasmas einer Glimmentladung. Z. Naturforsch. 30a, 216 (1975).

${ }^{134} \mathrm{P}$. A. Büger and S. El Alfy, Messung von Anregungstemperaturen in einer Glimmentladungslampe. Z. Naturforsch. 30a, 466 (1975).

${ }^{135} \mathrm{H}$. Meinel and L. Krauss, Über die Besetzung der Rotationszustände von $\mathrm{OH}_{\text {Hnd }} \mathrm{C}_{2}$ in Niederdruckplasmen. J. Quant. Spectr. Radiative Transfer 9, 443 (1969).

${ }^{136} \mathrm{M}$. Cacciatore and M. Capitelli, The temporal evolution of population densities of excited states in atomic oxygen optically thin plasmas. J. Quart. Spectr. Radiative Transfer 16, 325 (1976).

${ }^{137} \mathrm{H}$. W. Drawin and B. Dubreuil, Equilibrium between radiation and matter through multiphoton processes. Physica 82C (1976). In press.

${ }^{138} \mathrm{~J}$. Hackmann, H. Reuters and J. Uhlenbusch, Berechnung von Plasmaparameters in Wandnähe, in Proc. Workshop on Plasma-Wall Interactions, University of Düsseldorf, 2 July 1974, (edited by J. Hackmann and J. Uhlenbusch) Physics Institute II, University of Düsseldorf (1975). 